FERMILAB-Conf-92/313-A

\title{
INFLATION AFTER COBE Lectures on Inflationary Cosmology
}

\author{
Michael S. Turner \\ Departments of Physics and Astronomy 83 Astrophysics, \\ Enrico Fermi Institute, The University of Chicago, Chicago, IL 60637-1433 \\ NASA/Fermilab Astrophysics Center, \\ Fermi National Accelerator Laboratory, Batavia, IL 60510-0500
}

\begin{abstract}
In these lectures I review the standard hot big-bang cosmology, emphasizing its successes, its shortcomings, and its major challenge - a detailed understanding of the formation of structure in the Universe. I then discuss the motivations for - and the fundamentals of - inflationary cosmology, particularly emphasizing the quantum origin of metric (density and gravity-wave) perturbations. Inflation addresses the shortcomings of the standard cosmology and provides the "initial data" for structure formation. I conclude by addressing the implications of inflation for structure formation, evaluating the various cold dark matter models in the light of the recent detection of temperature anisotropies in the cosmic background radiation by COBE. In the near term, the study of structure formation offers a powerful probe of inflation, as well as specific inflationary models.
\end{abstract}

\section{Hot Big Bang: Successes and Challenges}

\subsection{Successes}

The hot big-bang model, more properly the Friedmann-Robertson-Walker (FRW) cosmology or standard cosmology, is spectacularly successful: In short, it provides a reliable and tested accounting of the history of the Universe from about $0.01 \mathrm{sec}$ after the bang until today, some 15 billion years later. The primary pieces of evidence that support the model are: (1) The expansion of the Universe; (2) The cosmic background radiation; and (3) The primordial abundances of the light elements D, ${ }^{3} \mathrm{He},{ }^{4} \mathrm{He}$, and ${ }^{7} \mathrm{Li}$ 伹.

*Summary of lectures given at TASI-92 (Boulder, CO, June 1992), Cargese Summer School on Quantitative Particle Physics (Cargese, Corsica, July 1992), and Varenna Summer School on Galaxy Formation (Varenna, Italy, July 1992). 
Figure 1: Hubble diagram (from [3]). The deviation from a linear relationship around $40 \mathrm{Mpc}$ is due to peculiar velocities.

\subsubsection{The expansion}

Although the precise value of the Hubble constant is not known to better than a factor of two, $H_{0}=100 h \mathrm{~km} \mathrm{sec}^{-1} \mathrm{Mpc}^{-1}$ with $h=0.4-1$, there is little doubt that the expansion obeys the "Hubble law" out to red shifts approaching unity [2, 3]; see Fig. 1. As is well appreciated, the fundamental difficulty in determining the Hubble constant is the calibration of the cosmic-distance scale as "standard candles" are required 4 , 5].

The Hubble law allows one to infer the distance to an object from its red shift $z$ : $d=z H_{0}^{-1} \simeq 3000 z h^{-1} \mathrm{Mpc}$ (for $z \ll 1$, the galaxy's recessional velocity $v \simeq z c$ ), and hence "maps of the Universe" constructed from galaxy positions and red shifts are referred to as red-shift surveys. Ordinary galaxies and clusters of galaxies are seen out to red shifts of order unity; more unusual and rarer objects, such as radio galaxies and quasars, are seen out to red shifts of almost five (the current record holder is a quasar with red shift 4.9). Thus, we can probe the Universe with visible light to within a few billion years of the big bang. 


\subsubsection{The cosmic background radiation}

The spectrum of the cosmic background radiation (CBR) is consistent that of a black body at temperature $2.73 \mathrm{~K}$ over more than three decades in wavelength $(\lambda \sim$ $0.03 \mathrm{~cm}-100 \mathrm{~cm}$ ); see Fig. 2. The most accurate measurement of the temperature and spectrum is that by the FIRAS instrument on the COBE satellite which determined its temperature to be $2.726 \pm 0.01 \mathrm{~K}$ [6]. It is difficult to come up with a process other than an early hot and dense phase in the history of the Universe that would lead to such a precise black body [7]. According to the standard cosmology, the surface of last scattering for the CBR is the Universe at a red shift of about 1100 and an age of about $180,000\left(\Omega_{0} h^{2}\right)^{-1 / 2}$ yrs. It is possible that the Universe became ionized again after this epoch, or due to energy injection never recombined; in this case the last-scattering surface is even "closer," $z_{\mathrm{LSS}} \simeq 10\left[\Omega_{B} h / \sqrt{\Omega_{0}}\right]^{-2 / 3}$.

The temperature of the CBR is very uniform across the sky, to better than a part in $10^{4}$ on angular scales from tens of arcseconds to 90 degrees; see Fig. 3. Three forms of temperature anisotropy - two spatial and one temporal - have now been detected: (1) A dipole anisotropy of about a part in $10^{3}$, generally believed to be due to the motion of galaxy relative to the cosmic rest frame, at a speed of about $620 \mathrm{~km} \mathrm{sec}^{-1}$ [9]; (2) A yearly modulation in the temperature in a given direction on the sky of about a part in $10^{4}$, due to our orbital motion around the sun at $30 \mathrm{~km} \mathrm{sec}^{-1}$, see Fig. 4 [10]; and (3) The temperature anisotropies detected by the Differential Microwave Radiometer (DMR) on the Cosmic Background Explorer (COBE) satellite, $\left\langle(\Delta T / T)^{2}\right\rangle_{10^{\circ}}^{1 / 2}=1.1 \pm 0.2 \times 10^{-5}$ and $(\Delta T / T)_{Q}=6 \pm 2 \times 10^{-6}$, where the first measurement refers to the rms temperature fluctuation averaged over the entire sky as measured by a beam of width $10^{\circ}$, and the second is the magnitude of the quadrupole temperature anisotropy [1]. The $10^{\circ}$ and quadrupole anisotropies provide strong evidence for primeval density inhomogeneities of the same magnitude, which amplified by gravity, grew into the structures that we see today: galaxies, clusters of galaxies, superclusters, voids, walls, etc.

\subsubsection{Primordial nucleosynthesis}

Last, but certainly not least, there are the abundance of the light elements. According to the standard cosmology, when the age of the Universe was measured in seconds, the temperatures were of order $\mathrm{MeV}$, and the conditions were right for nuclear reactions which ultimately led to the synthesis of significant amounts of $\mathrm{D},{ }^{3} \mathrm{He},{ }^{4} \mathrm{He}$, and ${ }^{7} \mathrm{Li}$. The yields of primordial nucleosynthesis depend upon the baryon density, quantified as the baryon-to-photon ratio $\eta$, and the number of very light $(\lesssim \mathrm{MeV}$ ) particle species, often quantified as the equivalent number of light neutrino species, $N_{\nu}$. The predictions for the primordial abundances of all four light elements agree with their measured abundances provided that $3 \times 10^{-10} \lesssim \eta \lesssim 5 \times 10^{-10}$ and $N_{\nu} \lesssim 3.4$; see Fig. 5 [15].

Accepting the success of the standard model of nucleosynthesis, our precise knowledge of the present temperature of the Universe allows us to convert $\eta$ to a mass 
Figure 2: (a) COBE FIRAS measurements of the CBR temperature; (b) Summary of other CBR temperature measurements (from [6]); the dotted curve indicates the data from the other high-precision measurement, by the UBC rocket-borne COBRA instrument [8]. 
Figure 3: Summary of recent high-sensitivity CBR anisotropy measurements; with the exception of COBE all results are upper limits (from [12, 13]). The solid boxes (MIT balloon experiment) have recently been reanalyzed and shown to be a detection which is consistent with the COBE DMR result [14]. 
Figure 4: Yearly modulation of the CBR temperature - the earth really orbits the sun(!) (from [10]). 
Figure 5: Predicted light-element abundances and inferred abundances (from [15]). The measured primordial abundances are indicated and the concordance region is shaded. 
density, and by dividing by the critical density, $\rho_{\text {crit }} \simeq 1.88 h^{2} \times 10^{-29} \mathrm{~g} \mathrm{~cm}^{-3}$, to the fraction of critical density contributed by ordinary matter:

$$
0.011 \lesssim \Omega_{B} h^{2} \lesssim 0.019 ; \quad \Rightarrow \quad 0.011 \lesssim \Omega_{B} \lesssim 0.12
$$

this is the most accurate determination of the baryon density. Note, the uncertainty in the value of the Hubble constant leads to most of the uncertainty in $\Omega_{B}$.

The nucleosynthesis bound to $N_{\nu}$, and more generally to the number of light degrees of freedom in thermal equilibrium at the epoch of nucleosynthesis, is consistent with precision measurements of the properties of the $Z^{0}$ boson, which give $N_{\nu}=3.0 \pm 0.05$; further, the cosmological bound predates these accelerator measurements! The nucleosynthesis bound provides a stringent limit to the existence of new, light particles (even beyond neutrinos), and even provides a bound to the mass the tau neutrino, excluding a tau-neutrino mass between $0.5 \mathrm{MeV}$ and $25 \mathrm{MeV}$ [16]. Primordial nucleosynthesis provides a beautiful illustration of the powers of the Heavenly Laboratory, though it is outside the focus of these lectures.

The remarkable success of primordial nucleosynthesis gives us confidence that the standard cosmology provides an accurate accounting of the Universe at least as early as $0.01 \mathrm{sec}$ after the bang, when the temperature was about $10 \mathrm{MeV}$.

\subsubsection{Et cetera - and the age crisis?}

There are additional lines of reasoning and evidence that support the standard cosmology [7]. I mention two: the age of the Universe and structure formation. I discuss the basics of structure formation a bit later; for now it suffices to say that the standard cosmology provides a basic framework for understanding the formation of structuregravitational instability — which has recently been confirmed by COBE [11]. Here I will focus on the age of the Universe.

The expansion age of the Universe - time back to zero size - depends upon the present expansion rate, energy content, and equation of state: $t_{\exp }=f(\rho, p) H_{0}^{-1} \simeq$ $9.8 h^{-1} f(\rho, p)$ Gyr. For a matter-dominated Universe, $f$ is between 1 and $2 / 3$ (for $\Omega_{0}$ between 0 and 1), so that the expansion age is somewhere between 7 Gyr and 20 Gyr. There are other independent measures of the age of the Universe, e.g., based upon long-lived radioisotopes, the oldest stars, and the cooling of white dwarfs. These "ages," ranging from 13 to $18 \mathrm{Gyr}$, span the same interval(!). This wasn't always the case; as late as the early 1950's it was believe that the Hubble constant was $500 \mathrm{~km} \mathrm{sec}^{-1} \mathrm{Mpc}^{-1}$, implying an expansion age of at most $2 \mathrm{Gyr}$ - less than the age of the earth. This discrepancy was an important motivation for the steady-state cosmology.

While there is general agreement between the expansion age and other determinations of the age of the Universe, some cosmologists are worried that cosmology is on the verge of another age crisis [5]. Let me explain, while Sandage and a few others continue to obtain values for the Hubble constant around $50 \mathrm{~km} \mathrm{~s}^{-1} \mathrm{Mpc}^{-1}$ [2], a variety of different techniques seem to be converging on a value around $80 \pm 10 \mathrm{~km} \mathrm{~s}^{-1} \mathrm{Mpc}^{-1}$ 
[5]. If $H_{0}=80 \mathrm{~km} \mathrm{~s}^{-1} \mathrm{Mpc}^{-1}$, then $t_{\exp }=12 f(\rho, p) \mathrm{Gyr}$, and for $\Omega_{0}=1, t_{\exp }=8 \mathrm{Gyr}$, which is clearly inconsistent with other measures of the age. If $H_{0}=80 \mathrm{~km} \mathrm{~s}^{-1} \mathrm{Mpc}^{-1}$, one is almost forced to consider the radical alternative of a cosmological constant. For example, even with $\Omega_{0}=0.2, f \simeq 0.85$, corresponding to $t_{\exp } \simeq 10 \mathrm{Gyr}$; on the other hand, for a flat Universe with $\Omega_{\Lambda}=0.8, f \simeq 1.1$ and the expansion age $t_{\text {exp }} \simeq 13.5$ Gyr. As I shall discuss later, structure formation provides another motivation for a cosmological constant. My own gut-level feeling is that when the dust settles, we will find that $H_{0}=50 \mathrm{~km} \mathrm{~s}^{-1} \mathrm{Mpc}^{-1}$; then again, maybe not.

\subsection{Basics of the Big Bang Model}

The standard cosmology is based upon the maximally spatially symmetric RobertsonWalker line element

$$
d s^{2}=d t^{2}-R(t)^{2}\left[\frac{d r^{2}}{1-k r^{2}}+r^{2}\left(d \theta^{2}+\sin ^{2} \theta d \phi^{2}\right)\right]
$$

where $R(t)$ is the cosmic-scale factor, $R_{\text {curv }} \equiv R(t)|k|^{-1 / 2}$ is the curvature radius, and $k /|k|=-1,0,1$ is the curvature signature. All three models are without boundary: the positively curved model is finite and "curves" back on itself; the negatively curved and flat models are infinite in extent (though finite versions of both can be constructed by imposing a periodic structure: identifying all points in space with a fundamental cube). The Robertson-Walker metric embodies the observed isotropy and homogeneity of the Universe. It is interesting to note that this form of the line element was originally introduced for sake of mathematical simplicity; we now know that it is well justified at early times or today on large scales ( $\gg 10 \mathrm{Mpc}$ ), at least within our Hubble volume.

The coordinates, $r, \theta$, and $\phi$, are referred to as comoving coordinates: A particle at rest in these coordinates remains at rest, i.e., constant $r, \theta$, and $\phi$. A freely moving particle eventually comes to rest these coordinates, as its momentum is red shifted by the expansion, $p \propto R^{-1}$. Motion with respect to the comoving coordinates (or cosmic rest frame) is referred to as peculiar velocity; unless "supported" by the inhomogeneous distribution of matter peculiar velocities decay away as $R^{-1}$. Thus the measurement of peculiar velocities, which is not easy as it requires independent measures of both the distance and velocity of an object, can be used to probe the distribution of mass in the Universe.

Physical separations (i.e., measured by meter sticks) between freely moving particles scale as $R(t)$; or said another way the physical separation between two points is simply $R(t)$ times the coordinate separation. The momenta of freely propagating particles decrease, or "red shift," as $R(t)^{-1}$, and thus the wavelength of a photon stretches as $R(t)$, which is the origin of the cosmological red shift. The red shift suffered by a photon emitted from a distant galaxy $1+z=R_{0} / R(t)$; that is, a galaxy whose light is red shifted by $1+z$, emitted that light when the Universe was a factor of $(1+z)^{-1}$ smaller. Thus, when the light from the most distant quasar yet seen 
$(z=4.9)$ was emitted the Universe was a factor of almost six smaller; when CBR photons last scattered the Universe was about 1100 times smaller.

\subsubsection{Friedmann equation and the First Law}

The evolution of the cosmic-scale factor is governed by the Friedmann equation

$$
H^{2} \equiv\left(\frac{\dot{R}}{R}\right)^{2}=\frac{8 \pi G \rho_{\mathrm{tot}}}{3}-\frac{k}{R^{2}} ;
$$

where $\rho_{\text {tot }}$ is the total energy density of the Universe, matter, radiation, vacuum energy, and so on. A cosmological constant is often written as an additional term (= $\Lambda / 3$ ) on the rhs; I will choose to treat it as a constant energy density ("vacuum-energy density"), where $\rho_{\mathrm{vac}}=\Lambda / 8 \pi G$. (My convention in this regard is not universal.) The evolution of the energy density of the Universe is governed by

$$
d\left(\rho R^{3}\right)=-p d R^{3}
$$

which is the First Law of Thermodynamics for a fluid in the expanding Universe. (In the case that the stress energy of the Universe is comprised of several, noninteracting components, this relation applies to each separately; e.g., to the matter and radiation separately today.) For $p=\rho / 3$, ultra-relativistic matter, $\rho \propto R^{-4}$; for $p=0$, very nonrelativistic matter, $\rho \propto R^{-3}$; and for $p=-\rho$, vacuum energy, $\rho=$ const. If the rhs of the Friedmann equation is dominated by a fluid with equation of state $p=\gamma \rho$, it follows that $\rho \propto R^{-3(1+\gamma)}$ and $R \propto t^{2 / 3(1+\gamma)}$.

We can use the Friedmann equation to relate the curvature of the Universe to the energy density and expansion rate:

$$
\frac{k / R^{2}}{H^{2}}=\Omega-1 ; \quad \Omega=\frac{\rho_{\text {tot }}}{\rho_{\text {crit }}}
$$

and the critical density today $\rho_{\text {crit }}=3 H^{2} / 8 \pi G=1.88 h^{2} \mathrm{~g} \mathrm{~cm}^{-3} \simeq 1.05 \times 10^{4} \mathrm{eV} \mathrm{cm}^{-3}$. There is a one to one correspondence between $\Omega$ and the spatial curvature of the Universe: positively curved, $\Omega_{0}>1$; negatively curved, $\Omega_{0}<1$; and flat $\left(\Omega_{0}=1\right)$. Further, the "fate of the Universe" is determined by the curvature: model universes with $k \leq 0$ expand forever, while those with $k>0$ necessarily recollapse. The curvature radius of the Universe is related to the Hubble radius and $\Omega$ by

$$
R_{\mathrm{curv}}=\frac{H^{-1}}{|\Omega-1|^{1 / 2}} .
$$

In physical terms, the curvature radius sets the scale for the size of spatial separations where the effects of curved space become "pronounced." And in the case of the positively curved model it is just the radius of the 3 -sphere.

The energy content of the Universe consists of matter and radiation (today, photons and neutrinos). Since the photon temperature is accurately known, $T_{0}=$ $2.73 \pm 0.01 \mathrm{~K}$, the fraction of critical density contributed by radiation is also accurately known: $\Omega_{\text {rad }} h^{2}=4.18 \times 10^{-5}$. The matter content is another matter. 


\subsubsection{A short diversion concerning the present mass density}

The matter density today, i.e., the value of $\Omega_{0}$, is not nearly so well known [17]. Stars contribute less than $1 \%$ of critical density; based upon nucleosynthesis, we can infer that baryons contribute between $1 \%$ and $10 \%$ of critical. The dynamics of various systems allow astronomers to infer their gravitational mass. With their telescopes they measure the amount of light, and form a mass-to-light ratio. Multiplying this by the measured luminosity density of the Universe gives a determination of the mass density. (The critical mass-to-light ratio is $1200 h M_{\odot} / \mathcal{L}_{\odot}$.)

The motions of stars and gas clouds in spiral galaxies indicate that most of the mass of spiral galaxies exists in the form of dark (i.e., no detectable radiation), extended halos, whose full extent is still not known. Many cite the flat rotation curves of spiral galaxies, which indicate that the halo density decreases as $r^{-2}$, as the best evidence that most of the matter in the Universe is dark. Taking the mass-to-light ratio inferred for spiral galaxies to be typical of the Universe as a whole and remembering that the full extent of the dark matter halos is not known, one infers $\Omega_{\text {halo }} \gtrsim 0.03-0.1$.

The masses of clusters of galaxies can be estimated using the virial theorem, and these mass estimates too indicate the presence of large amounts of dark matter. Taking cluster mass-to-light ratios to be typical of the Universe as a whole, in spite of the fact that only about 1 in 10 galaxies resides in a cluster, one infers $\Omega_{\text {cluster }} \sim$ $0.1-0.3$.

Most galaxies are found in associations of a few galaxies known as small groups. Estimating the masses of these systems using dynamics is tricky because of the problem of "interlopers," galaxies that happen to be in the same part of the sky, but are not associated with the group [18]. This Fall, however, ROSAT detected the weak x-ray emission from the hot gas in the small group NGC 2300 [19]; from their measurements they were able to infer the shape of the gravitational potential - and hence total mass of the group - as well as the mass of the x-ray emitting gas and the visible mass in galaxies. They found that the total mass of the group was about 20 times that in ordinary matter(!). If one takes this to be a universal ratio of the total amount of matter to that in baryons and $\Omega_{B} \sim 0.05$, one concludes that $\Omega_{0} \sim 1$.

Not one of these methods is wholly satisfactory: Rotation curves of spiral galaxies are still "flat" at the last measured points, indicating that the mass is still increasing; likewise, cluster virial mass estimates are insensitive to material that lies beyond the region occupied by the visible galaxies - and moreover, only about one galaxy in ten resides in a cluster. What one would like is a measurement of the mass of a very big sample of the Universe, say a cube of $100 h^{-1} \mathrm{Mpc}$ on a side, which contains tens of thousands of galaxies.

Over the past five years or so progress has been made toward such a measurement. It involves the peculiar motion of our own galaxy, at a speed of about $620 \mathrm{~km} \mathrm{sec}^{-1}$ in the general direction of Hydra-Centaurus. This motion is due to the lumpy distribution of matter in our vicinity. By using gravitational-perturbation theory (actually, not much more than Newtonian physics) and the distribution of galaxies in our vicin- 
ity (as determined by the IRAS catalogue of infrared selected galaxies), one can infer the average mass density in a very large volume and thereby $\Omega_{0}$.

The basic physics behind the method is simple: the net gravitational pull on our galaxy depends both upon how inhomogeneous the distribution of galaxies is and how much mass is associated with each galaxy; by measuring the distribution of galaxies and our peculiar velocity one can infer the "mass per galaxy" and $\Omega_{0}$.

The value that has been inferred is $\operatorname{big}(!): \Omega_{\text {IRAS }} \sim 1 \pm 0.2$ [20]. Moreover, the measured peculiar velocities of other galaxies in this volume, more than thousand, have been used in a similar manner and indicate a similarly large value for $\Omega_{0}$ [21]. While this technique is very powerful, it does have its drawbacks: One has to make simple assumptions about how accurately mass is traced by light (the observed galaxies); one has to worry whether or not a significant portion of our galaxy's velocity is due to galaxies outside the IRAS sample - if so, this would lead to an overestimate of $\Omega_{0}$; and so on. This technique is not only very promising - but provides the "correct" answer (in my opinion!).

The so-called classical kinematic tests-Hubble diagram, angle-red shift relation, galaxy count-red shift relation - can, in principle, provide a determination of $\Omega_{0}$ [22]. However, all these methods require standard candles, rulers, or galaxies, and for this reason have proved inconclusive. However, that hasn't stopped efforts to use these tests, particularly the galaxy number-count test [23], and one or more of these classical tests may one day provide a definitive measurement.

To summarize this aside on the mass density of the Universe:

1. Most of the matter is dark.

2. Baryons provide between about $1 \%$ and $10 \%$ of the mass density.

3. $\Omega_{0}$ could conceivably be as small as 0.1 - in which case all the dark matter could be baryons (e.g., neutron stars, "jupiters," and so on).

4. If asked for the value of $\Omega_{0}$, a typical astronomer would respond with a number in the interval $0.2 \pm 0.1$.

5. The evidence continues to mount for a gap between $\Omega_{B}$ and $\Omega_{0}$-in which case nonbaryonic dark matter is required.

The current prejudice - and certainly that of this author - is a flat Universe $\left(\Omega_{0}=\right.$ 1 ) with nonbaryonic dark matter, $\Omega_{X} \sim 1 \gg \Omega_{B}$. However, I shall continue to display the $\Omega_{0}$ dependence of important quantities.

\subsubsection{The early, radiation-dominated Universe}

In any case, at present, matter outweighs radiation by a wide margin. However, since the energy density in matter decreases as $R^{-3}$, and that in radiation as $R^{-4}$ (the extra factor due to the red shifting of the energy of relativistic particles), at early 
times the Universe was radiation dominated - indeed the calculations of primordial nucleosynthesis provide excellent evidence for this. Denoting the epoch of matterradiation equality by subscript 'EQ,' and using $T_{0}=2.73 \mathrm{~K}$, it follows that

$$
\begin{gathered}
R_{\mathrm{EQ}}=4.18 \times 10^{-5}\left(\Omega_{0} h^{2}\right)^{-1} ; \quad T_{\mathrm{EQ}}=5.62\left(\Omega_{0} h^{2}\right) \mathrm{eV} ; \\
t_{\mathrm{EQ}}=4.17 \times 10^{10}\left(\Omega_{0} h^{2}\right)^{-2} \mathrm{sec} .
\end{gathered}
$$

At early times the expansion rate and age of the Universe were determined by the temperature of the Universe and the number of relativistic degrees of freedom:

$$
\begin{gathered}
\rho_{\mathrm{rad}}=g_{*}(T) \frac{\pi^{2} T^{4}}{30} ; \quad H \simeq 1.67 g_{*}^{1 / 2} T^{2} / m_{\mathrm{Pl}} \\
\Rightarrow R \propto t^{1 / 2} ; \quad t \simeq 2.42 \times 10^{-6} g_{*}^{-1 / 2}(T / \mathrm{GeV})^{-2} \mathrm{sec} ;
\end{gathered}
$$

where $g_{*}(T)$ counts the number of ultra-relativistic degrees of freedom $(\approx$ the sum of the internal degrees of freedom of particle species much less massive than the temperature) and $m_{\mathrm{Pl}} \equiv G^{-1 / 2}=1.22 \times 10^{19} \mathrm{GeV}$ is the Planck mass. For example, at the epoch of nucleosynthesis, $g_{*}=10.75$ assuming three, light ( $\left.\ll \mathrm{MeV}\right)$ neutrino species; taking into account all the species in the standard model, $g_{*}=106.75$ at temperatures much greater than $300 \mathrm{GeV}$; see Fig. 6 .

A quantity of importance related to $g_{*}$ is the entropy density in relativistic particles,

$$
s=\frac{\rho+p}{T}=\frac{2 \pi^{2}}{45} g_{*} T^{3},
$$

and the entropy per comoving volume,

$$
S \propto R^{3} s \propto g_{*} R^{3} T^{3} .
$$

By a wide margin most of the entropy in the Universe exists in the radiation bath. The entropy density is proportional to the number density of relativistic particles. At present, the relativistic particle species are the photons and neutrinos, and the entropy density is a factor of 7.04 times the photon-number density: $n_{\gamma}=413 \mathrm{~cm}^{-3}$ and $s=2905 \mathrm{~cm}^{-3}$.

In thermal equilibrium - which provides a good description of most of the history of the Universe - the entropy per comoving volume $S$ remains constant. This fact is very useful. First, it implies that the temperature and scale factor are related by

$$
T \propto g_{*}^{-1 / 3} R^{-1}
$$

which for $g_{*}=$ const leads to the familiar $T \propto R^{-1}$.

Second, it provides a way of quantifying the net baryon number (or any other particle number) per comoving volume:

$$
N_{B} \equiv R^{3} n_{B}=\frac{n_{B}}{s} \simeq(4-7) \times 10^{-11}
$$


Figure 6: The total effective number of relativistic degrees of freedom $g_{*}(T)$ in the standard model of particle physics as a function of temperature. 
The baryon number of the Universe tells us two things: (1) the entropy per particle in the Universe is extremely high, about $10^{10}$ or so compared to about $10^{-2}$ in the sun and a few in the core of a newly formed neutron star. (2) The asymmetry between matter and antimatter is very small, about $10^{-10}$, since at early times quarks and antiquarks were roughly as abundant as photons. One of the great successes of particle cosmology is baryogenesis, the idea that $B, C$, and $C P$ violating interactions occurring out-of-equilibrium early on allow the Universe to develop a net baryon number of this magnitude [24].

Finally, the constancy of the entropy per comoving volume allows us to characterize the size of comoving volume corresponding to our present Hubble volume in a very physical way: by the entropy it contains,

$$
S_{U}=\frac{4 \pi}{3} H_{0}^{-3} s \simeq 10^{90}
$$

\subsubsection{The earliest history}

The standard cosmology is tested back to times as early as about 0.01 sec; it is only natural to ask how far back one can sensibly extrapolate. Since the fundamental particles of Nature are point-like quarks and leptons whose interactions are perturbatively weak at energies much greater than $1 \mathrm{GeV}$, one can imagine extrapolating as far back as the epoch where general relativity becomes suspect, i.e., where quantum gravitational effects are likely to be important: the Planck epoch, $t \sim 10^{-43}$ sec and $T \sim 10^{19} \mathrm{GeV}$. Of course, at present, our firm understanding of the elementary particles and their interactions only extends to energies of the order of $100 \mathrm{GeV}$, which corresponds to a time of the order of $10^{-11} \mathrm{sec}$ or so. We can be relatively certain that at a temperature of $100 \mathrm{MeV}-200 \mathrm{MeV}\left(t \sim 10^{-5} \mathrm{sec}\right)$ there was a transition (likely a second-order phase transition) from quark/gluon plasma to very hot hadronic matter, and that some kind of phase transition associated with the symmetry breakdown of the electroweak theory took place at a temperature of the order of $300 \mathrm{GeV}$ $\left(t \sim 10^{-11} \mathrm{sec}\right)$.

It is interesting to look at the progress that has taken place since Weinberg's classic text on cosmology was published in 1972 [25]; at that time many believed that the Universe had a limiting temperature of the order of several hundred $\mathrm{MeV}$, due to the exponentially rising number of particle states, and that one could not speculate about earlier times. Today, based upon our present knowledge of physics and powerful mathematical tools (e.g., gauge theories, grand unified theories, and superstring theory) we are able to make quantitative speculations back to the Planck epoch - and even earlier. Of course, these speculations could be totally wrong, based upon a false sense of confidence (arrogance?). As I shall discuss, inflation is one of these well defined-and well motivated-speculations about the history of the Universe well after the Planck epoch, but well before primordial nucleosynthesis. 


\subsubsection{The matter and curvature dominated epochs}

After the equivalence epoch, the matter density exceeds that of radiation. During the matter-dominated epoch the scale factor grows as $t^{2 / 3}$ and the age of the Universe is related to red shift by

$$
t=2.06 \times 10^{17}\left(\Omega_{0} h^{2}\right)^{-1 / 2}(1+z)^{-3 / 2} \text { sec. }
$$

If $\Omega_{0}<1$, the matter-dominated epoch is followed by a "curvature-dominated" epoch where the rhs of the Friedmann equation is dominated by the $|k| / R^{2}$ term. When the Universe is curvature dominated it is said to expand freely, no longer decelerating since the gravitational effect of matter has become negligible: $\ddot{R} \approx 0$ and $R \propto t$. The epoch of curvature dominance begins when the matter and curvature terms are equal:

$$
R_{\mathrm{CD}}=\frac{\Omega_{0}}{1-\Omega_{0}} \longrightarrow \Omega_{0} ; \quad z_{\mathrm{CD}}=\Omega_{0}^{-1}-2 \longrightarrow \Omega_{0}^{-1}
$$

where the limits shown are for $\Omega_{0} \rightarrow 0$. By way of comparison, in a flat Universe with a cosmological constant, the Universe becomes "vacuum dominated" when $R=R_{\text {vac }}$ :

$$
R_{\mathrm{vac}}=\left(\frac{\Omega_{0}}{1-\Omega_{0}}\right)^{1 / 3} \longrightarrow \Omega_{0}^{1 / 3} ; \quad z_{\mathrm{vac}}=\left(\frac{1-\Omega_{0}}{\Omega_{0}}\right)^{1 / 3}-1 \longrightarrow \Omega_{0}^{-1 / 3}
$$

For a given value of $\Omega_{0}$, the transition occurs much more recently, which has important implications for structure formation since small density perturbations only grow during the matter-dominated era.

\subsubsection{One last thing: horizons}

In spite of the fact that the Universe was vanishingly small at early times, the rapid expansion precluded causal contact from being established throughout. Photons travel on null paths characterized by $d r=d t / R(t)$; the physical distance that a photon could have traveled since the bang until time $t$, the distance to the horizon, is

$$
\begin{aligned}
d_{H}(t) & =R(t) \int_{0}^{t} \frac{d t^{\prime}}{R\left(t^{\prime}\right)} \\
& =t /(1-n)=n H^{-1} /(1-n) \quad \text { for } R(t) \propto t^{n}, \quad n<1 .
\end{aligned}
$$

Note, in the standard cosmology the distance to the horizon is finite, and up to numerical factors, equal to the age of the Universe or the Hubble radius, $H^{-1}$. For this reason, I will use horizon and Hubble radius interchangeably.?

\footnotetext{
${ }^{1}$ In inflationary models the horizon and Hubble radius are not roughly equal as the horizon distance grows exponentially relative to the Hubble radius; in fact, at the end of inflation they differ by $e^{N}$, where $N$ is the number of e-folds of inflation. However, I will slip and use "horizon" and "Hubble radius" interchangeably, though I will always mean Hubble radius.
} 
An important quantity is the entropy within a horizon volume: $S_{\mathrm{HOR}} \sim H^{-3} T^{3}$; during the radiation-dominated epoch $H \sim T^{2} / m_{\mathrm{Pl}}$, so that

$$
S_{\mathrm{HOR}} \sim\left(\frac{m_{\mathrm{Pl}}}{T}\right)^{3}
$$

from this we conclude that at early times the comoving volume that encompasses all that we can see today (characterized by an entropy of $10^{90}$ ) was comprised of a very large number of causally disconnected regions.

\subsection{The challenge: development of structure}

This brings us to what I believe is the major challenge of the standard cosmology at present: a detailed understanding of the formation of structure in the Universe. We have every indication that the Universe at early times, say $t \ll 300,000 \mathrm{yrs}$, was very homogeneous; however, today inhomogeneity (or structure) is ubiquitous: stars $\left(\delta \rho / \rho \sim 10^{30}\right)$, galaxies $\left(\delta \rho / \rho \sim 10^{5}\right)$, clusters of galaxies $\left(\delta \rho / \rho \sim 10-10^{3}\right)$, superclusters, or "clusters of clusters" $(\delta \rho / \rho \sim 1)$, voids $(\delta \rho / \rho \sim-1)$, great walls, and so on.

For some 25 years the standard cosmology has provided a general framework for understanding this: Once the Universe becomes matter dominated (around 1000 yrs after the bang) primeval density inhomogeneities $\left(\delta \rho / \rho \sim 10^{-5}\right)$ are amplified by gravity and grow into the structure we see today [26]. The fact that a fluid of self-gravitating particles is unstable to the growth of small inhomogeneities was first pointed out by Jeans and is known as the Jeans instability. The existence of these inhomogeneities was confirmed in spectacular fashion by the COBE DMR discovery of CBR anisotropy this past spring: The temperature anisotropies detected almost certainly owe their existence to primeval density inhomogeneities, as causality precludes microphysical processes from producing anisotropies on angular scales larger than about $1^{\circ}$, the angular size of the horizon at last scattering.

At last, the basic picture has been put on firm ground (whew!). Now the challenge is to fill in the details - origin of the density perturbations, precise evolution of the structure, and so on. As I shall emphasize, such an understanding may well be within reach, and offers a window on the early Universe.

\subsubsection{The general picture: gravitational instability}

Let us begin by expanding the perturbation to the matter density in plane waves

$$
\frac{\delta \rho_{M}(\mathbf{x}, t)}{\rho_{M}}=\frac{1}{(2 \pi)^{3}} \int d^{3} k \delta_{k}(t) e^{-i \mathbf{k} \cdot \mathbf{x}},
$$

where $\lambda=2 \pi / k$ is the comoving wavelength of the perturbation and $\lambda_{\text {phys }}=R \lambda$ is the physical wavelength. The comoving wavelengths of perturbations corresponding to 
bright galaxies, clusters, and the present horizon scale are respectively: about $1 \mathrm{Mpc}$, $10 \mathrm{Mpc}$, and $3000 h^{-1} \mathrm{Mpc}$, where $1 \mathrm{Mpc} \simeq 3.09 \times 10^{24} \mathrm{~cm} \simeq 1.56 \times 10^{38} \mathrm{GeV}^{-1}$.

The growth of small matter inhomogeneities of wavelength smaller than the Hubble scale $\left(\lambda_{\text {phys }} \lesssim H^{-1}\right)$ is governed by a Newtonian equation:

$$
\ddot{\delta}_{k}+2 H \dot{\delta}_{k}+v_{s}^{2} k^{2} \delta_{k} / R^{2}=4 \pi G \rho_{M} \delta_{k},
$$

where $v_{s}^{2}=d p / d \rho_{M}$ is the square of the sound speed. Competition between the pressure term and the gravity term on the rhs determine whether or not pressure can counteract gravity: Perturbations with wavenumber larger than the Jeans wavenumber, $k_{J}^{2}=4 \pi G R^{2} \rho_{M} / v_{s}^{2}$, are Jeans stable and just oscillate; perturbations with smaller wavenumber are Jeans unstable and can grow. For cold dark matter $v_{s} \simeq 0$ and all scales are Jeans unstable; even for baryonic matter, after decoupling $k_{J}$ corresponds to a baryon mass of only about $10^{5} M_{\odot}$. All the scales of interest here are Jeans unstable and we will ignore the pressure term.

Let us discuss solutions to this equation under different circumstances. First, consider the Jeans problem, evolution of perturbations in a static fluid, i.e., $H=0$. In this case Jeans unstable perturbations grow exponentially, $\delta_{k} \propto \exp (t / \tau)$ where $\tau=$ $1 / \sqrt{4 G \pi \rho_{M}}$. Next, consider the growth of Jeans unstable perturbations in a matterdominated Universe, i.e., $H^{2}=8 \pi G \rho_{M} / 3$ and $R \propto t^{2 / 3}$. Because the expansion tends to "pull particles away from one another," the growth is only power law, $\delta_{k} \propto t^{2 / 3}$; i.e., at the same rate as the scale factor. Finally, consider a radiation or curvature dominated Universe, i.e., $8 \pi G \rho_{\mathrm{rad}} / 3$ or $|k| / R^{2}$ much greater than $8 \pi G \rho_{M} / 3$. In this case, the expansion is so rapid that matter perturbations grow very slowly, as $\ln R$ in radiation-dominated epoch, or not at all $\delta_{k}=$ const in the curvature-dominated epoch.

The growth of nonlinear perturbations is another matter; once a perturbation reaches an overdensity of order unity or larger it "separates" from the expansioni.e., becomes its own self-gravitating system and ceases to expand any further. In the process of virial relaxation, its size decreases by a factor of two - density increases by a factor of 8 ; thereafter, its density contrast grows as $R^{3}$ since the average matter density is decreasing as $R^{-3}$, though smaller scales could become Jeans unstable and collapse further to form smaller objects of higher density, stars, etc.

From this we learn that structure formation begins when the Universe becomes matter dominated and ends when it becomes curvature dominated (at least the growth of linear perturbations). The total growth available for linear perturbations is $R_{\mathrm{CD}} / R_{\mathrm{EQ}} \simeq 2.4 \times 10^{4} \Omega_{0}^{2} h^{2}$; since nonlinear structures have evolved by the present epoch, we can infer that primeval perturbations of the order $\left(\delta \rho_{M} / \rho_{M}\right)_{\mathrm{EQ}} \sim$ $4 \times 10^{-5}\left(\Omega_{0} h\right)^{-2}$ are required. Note that in a low-density Universe larger initial perturbations are necessary as there is less time for growth ("the low $\Omega_{0}$ squeeze"). Further, in a baryon-dominated Universe things are even more difficult as perturbations in the baryons cannot begin to grow until after decoupling since matter is tightly coupled to the radiation. (In a flat, low- $\Omega_{0}$ model with a cosmological constant the growth of linear fluctuations continues until almost today since $z_{\Lambda} \sim \Omega_{0}^{-1 / 3}$, and so the total growth factor is about $2.4 \times 10^{4}\left(\Omega_{0} h^{2}\right)$. We will return to this model later.) 


\subsubsection{CBR temperature fluctuations}

The existence of density inhomogeneities has another important consequence: fluctuations in the temperature of the CBR of a similar amplitude [27]. The temperature difference measured between two points separated by a large angle $\left(\gtrsim 1^{\circ}\right)$ arises due to a very simple physical effect:2 The difference in the gravitational potential between the two points on the last-scattering surface, which in turn is related to the density perturbation, determines the temperature anisotropy on the angular scale subtended by that length scale,

$$
\left(\frac{\delta T}{T}\right)_{\theta}=-\left(\frac{\delta \phi}{3}\right)_{\lambda} \approx \frac{1}{2}\left(\frac{\delta \rho}{\rho}\right)_{\mathrm{HOR}, \lambda}
$$

where the scale $\lambda \sim 100 h^{-1} \mathrm{Mpc}(\theta / \mathrm{deg})$ subtends an angle $\theta$ on the last-scattering surface. This is known as the Sachs-Wolfe effect [28].

The quantity $(\delta \rho / \rho)_{\mathrm{HOR}, \lambda}$ is the amplitude with which a density perturbation crosses inside the horizon, i.e., when $R \lambda \sim H^{-1}$. Since the fluctuation in the gravitational potential $\delta \phi \sim\left(R \lambda / H^{-1}\right)^{2}(\delta \rho / \rho)$, the horizon-crossing amplitude is equal to the gravitational potential (or curvature) fluctuation. The horizon-crossing amplitude $(\delta \rho / \rho)_{\text {HOR }}$ has several nice features: (i) during the matter-dominated era the potential fluctuation on a given scale remains constant, and thus the potential fluctuations at decoupling on scales that crossed inside the horizon after matter-radiation equality, corresponding to angular scales $\lesssim 0.1^{\circ}$, are just given by their horizon-crossing amplitude; (ii) because of its relationship to $\delta \phi$ it provides a dimensionless, geometrical measure of the size of the density perturbation on a given scale, and its effect on the CBR; (iii) by specifying perturbation amplitudes at horizon crossing one can effectively avoid discussing the evolution of density perturbations on scales larger than the horizon, where a Newtonian analysis does not suffice and where gauge subtleties (associated with general relativity) come into play; and finally (iv) the density perturbations generated in inflationary models are characterized by $(\delta \rho / \rho)_{\mathrm{HOR}} \simeq$ const.

On angular scales smaller than about $1^{\circ}$ two other physical effects lead to CBR temperature fluctuations: the motion of the last-scattering surface (Doppler) and the intrinsic fluctuations in the local photon temperature. These fluctuations are much more difficult to compute, and depend on microphysics - the ionization history of the Universe and the damping of perturbations in the photon-baryon fluid due to photon streaming. Not only are the Sachs-Wolfe fluctuations simpler to compute, but they accurately mirror the primeval fluctuations since at the epoch of decoupling microphysics is restricted to angular scales less than about a degree.

In sum, on large angular scales the Sachs-Wolfe effect dominates; on the scale of about $1^{\circ}$ the total CBR fluctuation is about twice that due to the Sachs-Wolfe effect; on smaller scales the Doppler and intrinsic fluctuations dominate. CBR temperature

\footnotetext{
${ }^{2}$ Large angles mean those larger than the angle subtended by the horizon-scale at decoupling, $\theta \sim H_{\mathrm{DEC}}^{-1} / H_{0}^{-1} \sim z_{\mathrm{DEC}}^{-1 / 2} \sim 1^{\circ}$.
} 
fluctuations on scales smaller than about $0.1^{\circ}$ are severely reduced by the smearing effect of the finite thickness of last-scattering surface.

Details aside, in the context of the gravitational instability scenario density perturbations of sufficient amplitude to explain the observed structure lead to temperature fluctuations in the CBR of characteristic size,

$$
\frac{\delta T}{T} \approx 10^{-5}\left(\Omega_{0} h\right)^{-2}
$$

To be sure I have brushed over important details, but this equation conveys a great deal. First, the overall amplitude is set by the inverse of the growth factor, which is just the ratio of the radiation energy density to matter density at present. Next, it explains why theoretical cosmologists were so relieved when the COBE DMR detected temperature fluctuations of this amplitude, and conversely why one heard offhanded remarks before the COBE DMR detection that the standard cosmology was in trouble because the CBR temperature was too uniform to allow for the observed structure to develop. Finally, it illustrates one of the reasons why cosmologists who study structure formation have embraced the flat-Universe model with such enthusiasm: If we accept the Universe that meets the eye, $\Omega_{0} \sim 0.1$ and baryons only, then the simplest models of structure formation predict temperature fluctuations of the order of $10^{-3}$, far too large to be consistent with observation. Later, I will mention Peebles' what-you-see-is-what-you-get model [29], also known as PIB for primeval baryon isocurvature fluctuation, which is still viable because the spectrum of perturbations decreases rapidly with scale so that the perturbations that give rise to CBR fluctuations are small (which is no mean feat). Historically, it was fortunate that one started with a low- $\Omega_{0}$, baryon-dominated Universe: the theoretical predictions for the CBR fluctuations were sufficiently favorable that experimentalist were stirred to try to measure them - and then, slowly, theorists lowered their predictions. Had

the theoretical expectations begun at $10^{-5}$, experimentalists might have been too discouraged to even try!

\subsubsection{An initial data problem}

With the COBE DMR detection in hand we can praise the success of the gravitational instability scenario; however, the details now remain to be filled in. The structure formation problem is now one of initial data, namely

1. The quantity and composition of matter in the Universe, $\Omega_{0}, \Omega_{B}$, and $\Omega_{\text {other }}$.

2. The spectrum of initial density perturbations: for the purist, $(\delta \rho / \rho)_{\mathrm{EQ}}$, or for the simulator, the Fourier amplitudes at the epoch of matter-radiation equality.

In a statistical sense, these initial data provide the "blueprint" for the formation of structure.

The initial data are the challenge and the opportunity. Although the gravitational instability picture has been around since the discovery of the CBR itself, the lack of 
specificity in initial data has impeded progress. With the advent of the serious study of the earliest history of the Universe a new door was opened. We now have several well motivated early-Universe blueprints: Inflation-produced density perturbations and nonbaryonic dark matter; cosmic-string produced perturbations and nonbaryonic dark matter [30]; texture produced density perturbations and nonbaryonic dark matter [31]; a baryon-dominated Universe with isocurvature fluctuations] [29]. Structure formation also provides the opportunity to probe the earliest history of the Universe, by testing these interesting, if not bold, blueprints. I will be focusing on the blueprints motivated by inflation.

\section{Inflation: An Overview}

\subsection{Shortcomings of the Standard Cosmology}

By now the shortcomings of the standard cosmology are well appreciated: the horizon or large-scale smoothness problem; the small-scale inhomogeneity problem (origin of density perturbations); the flatness or oldness problem; and the monopole problem. I will only briefly review them here. They do not indicate any logical inconsistencies of the standard cosmology; rather, that very special initial data seem to be required for evolution to a universe that is qualitatively similar to ours today. Nor is inflation the first attempt to address these shortcomings: Over the past two decades cosmologists have pondered this question and proposed other solutions [33]. Inflation is a solution based upon well-defined, albeit speculative, early-Universe microphysics describing the post-Planck epoch.

The uniformity of the CBR temperature, to better than a part in $10^{4}$, implies that the Universe on the largest scales (say $\gtrsim 100 h^{-1} \mathrm{Mpc}$ ) is very smooth as density inhomogeneities induce temperature fluctuations of a similar magnitude. The existence of particle horizons in the standard cosmology precludes explaining the smoothness as a result of microphysical events: The horizon at decoupling, the last time one could imagine temperature fluctuations being smoothed by particle interactions, corresponds to an angular scale on the sky of about $1^{\circ}$, which precludes temperature variations on larger scales from being erased. In terms of entropy, the presently observed Universe, corresponds to a comoving volume containing an entropy of order $10^{90}$; during the early radiation dominated epoch the horizon volume contained an entropy of order $\left(m_{\mathrm{Pl}} / T\right)^{3}$, implying that at early times our current Hubble volume consisted of countless, causally distinct regions.

To account for the small-scale lumpiness of the Universe today, density perturba-

\footnotetext{
${ }^{3}$ Isocurvature baryon-number fluctuations correspond at early times to fluctuations in the local baryon number but not the energy density. At late times, when the Universe is matter dominated, they become fluctuations in the mass density of a comparable amplitude.
} 
tions with horizon-crossing amplitudes of $10^{-5}$ on scales of $1 \mathrm{Mpc}$ to $10^{4} \mathrm{Mpc}$ or so are required. As can be seen in Fig. 7, in the standard cosmology the physical size of a perturbation, which grows as the scale factor, begins larger than the horizon and relatively late in the history of the Universe crosses inside the horizon,

$$
\begin{aligned}
t_{\mathrm{HOR}} & \simeq 3 \times 10^{8}(\lambda / \mathrm{Mpc})^{2} \mathrm{sec} & & \lambda \lesssim 13 h^{-2} \mathrm{Mpc} \\
& \simeq 3 \times 10^{7}(\lambda / \mathrm{Mpc})^{3} \mathrm{sec} & & \lambda \gtrsim 13 h^{-2} \mathrm{Mpc}
\end{aligned}
$$

This precludes a causal microphysical explanation for the origin of the required density perturbations.'t

The fact that $\Omega_{0}$ is of order unity means that the curvature radius is comparable to the Hubble radius. Had that been the case at the initial epoch, the Universe would be a very different place today: Since the curvature term in the Friedmann equation decreases only as $R^{-2}$, while the matter and radiation densities decrease as $R^{-3}$ and $R^{-4}$ respectively, a curvature radius comparable to the Hubble radius early on would have led to a Universe that quickly became curvature dominated. For positive curvature, recollapse would follow quickly, and for negative curvature, a coasting phase that would lead to a Universe that cools too quickly (for $t_{\text {initial }} \sim 10^{-43} \mathrm{sec}$, the temperature reaches $3 \mathrm{~K}$ at an age of $10^{-11} \mathrm{sec}$ ). Put another way, $\Omega$ is an unstable fixed point:

$$
\begin{aligned}
& \Omega(t)=\frac{1}{1-x(t)} \\
& x(t)=\frac{k / R^{2}}{8 \pi G \rho / 3} ;
\end{aligned}
$$

the deviation of $\Omega(t)$ from unity increases as $x(t) \propto R^{n}, n=2$ (radiation-dominated epoch), $n=1$ (matter-dominated epoch). In order that $\Omega$ still be close to unity today, it must have extremely close to unity early on; for $t_{\text {initial }} \sim 10^{-43} \mathrm{sec},\left|\Omega\left(t_{\text {initial }}\right)-1\right| \lesssim$ $10^{-60}$ is necessary. Thus, for most of its history the Universe must have been extremely flat, i.e., $R_{\text {curv }} \gg H^{-1}$; if $\Omega_{0}$ is not equal to unity, then the Universe just today is beginning to exhibit its curvature. Why now?

Last, I mention the monopole problem: The simplest grand unified theories and the standard cosmological lead to a disastrous prediction, the extreme overproduction of magnetic monopoles [34]. This overproduction traces to the smallness of the horizon at very early times: magnetic monopoles are produced as defects of the GUT phase transition at an abundance of about 1 per horizon volume which corresponds to a present monopole to photon ratio of order $\left(T_{\mathrm{GUT}} / m_{\mathrm{Pl}}\right)^{3}$.

\footnotetext{
${ }^{4} \mathrm{Of}$ course, it is possible to produce the perturbations at very late times, when the relevant scale has already crossed inside the horizon [32]; the motivation for the nonstandard microphysics required to do so is lacking at present. It is also possible for microphysics to produce isocurvature perturbations by producing a pressure wave that eventually propagates to large scales; this is the type of perturbation that is generated by cosmic strings or textures.
} 
Figure 7: The physical wavelength of a density perturbation and the horizon size $H^{-1}$ as a function of scale factor; $\lambda_{\mathrm{GAL}}$ indicates a galactic sized perturbation $(\lambda \sim 1 \mathrm{Mpc})$ and $\lambda_{\text {HOR }}$ corresponds to the present Hubble radius (horizon). Microphysics operates on scales $\lesssim H^{-1}$; without inflation scales cross the Hubble radius but once. 
The first three problems do not involve logical inconsistencies: The initial data for a perturbed FRW model that is extremely flat exist. Rather, it is the fact that such initial data are "very special" which is disturbing. Collins and Hawking quantified it: The set of initial data that evolve to a state qualitatively similar to our Universe is of measure zero [35]. Maybe the Creator had a lucky day! Or better yet, perhaps the present state of the Universe traces to events that took place early on. Inflation provides an interesting example of the latter.

\subsection{Generic Aspects of Inflation}

Inflationary cosmology has become a very mature subject in the decade since Guth wrote his influential paper [36] that launched the inflationary cosmology boom. While there are a multitude of different kinds of inflation (see below), two features are common to all models of inflation [37]

- Superluminal expansion

- Massive entropy production

Superluminal expansion refers to accelerated growth of the scale factor $(\ddot{R}>0$ which implies $R \propto t^{n}$ with $n>1$ ), and its necessity is easy to understand. In order that the physical size of a comoving scale, $d_{\text {phys }} \propto R(t)$, begin sub-Hubble size and and become super-Hubble size, $R(t)$ must increase faster than $t$ since $H^{-1} \propto R(t)^{1 / n}$. Thus, "superluminal" expansion is a necessary kinematic requirement if one is to both solve the horizon and create density perturbations (see Fig. 7).

The reason for the second requirement is equally simple: In the absence of entropy production the entropy per comoving volume $S \propto(R T)^{3}$ remains constant; rapid expansion can create a "very large" smooth patch, but the entropy within that patch remains constant. As discussed above, at early times the entropy within a horizonsized patch is very small, too small to account for the entropy within our present Hubble volume. Only massive entropy production can change this [37.

To illustrate, consider Guth's original model of inflation based upon a first-order phase transition [36]. The basic idea is that the Higgs field responsible for the spontaneous breakdown of the GUT symmetry gets "hung up" in a local, high-energy, minimum of its potential (more precisely, free-energy density). At high temperatures the state of minimum free energy is characterized by $\phi=0$, indicating that the full GUT symmetry is manifest; as the temperature drops below the critical temperature, the state of minimum free energy is characterized by $\phi \neq 0$, the state that exhibits broken symmetry. In a first-order transition $\phi=0$ can remain a local minimum of the free energy, separated from the global minimum by a potential barrier; see Fig. 8. During the time that $\phi$ is hung up the large vacuum-energy density, $\rho=V(\phi=0) \equiv \mathcal{M}^{4}$, drives very rapid expansion $(\mathcal{M}$ is the energy scale that characterizes of the symmetry breaking). 
Figure 8: The free-energy density as a function of temperature for a first-order phase transition.

For definiteness, take $\mathcal{M}=10^{14} \mathrm{GeV}$, a typical scale for inflation; the Hubble time associated with the false-vacuum energy $H^{-1} \sim 10^{-34}$ sec. The size of a region that one might expect to be smooth is of order $c t \sim 10^{-23} \mathrm{~cm}$; the entropy within such a patch is of order $10^{14}$. While the Higgs field is trapped in the false vacuum, the temperature of the Universe continues to decrease as $R^{-1}$; very soon the thermal energy density becomes insignificant compared to the constant false-vacuum energy density. At this point, the Universe enters a de Sitter phase of exponential expansion since $\rho \sim \mathcal{M}^{4}=$ const; this is the superluminal expansion. As the Universe expands, it cools exponentially with the entropy per comoving volume remaining constant; the smooth horizon-sized patch continues to contain an entropy of only $10^{14}$ as it grows exponentially in size.

During inflation the scale factor undergoes many e-folds; the precise number is determined by how long the Higgs field is hung up: $N=H \Delta t$. Again, for definiteness, suppose that the Universe gets hung up for a mere $10^{-32}$ sec; then, during inflation the patch grows in linear size by $e^{100} \sim 10^{43}$ and its temperature drops by the same factor. Thus far, inflation has done little. When the Higgs field does make its way to the true vacuum, the enormous false-vacuum energy is released and ultimately thermalized, reheating the patch to a temperature of order $\mathcal{M} \sim 10^{14} \mathrm{GeV}$, thereby increasing the entropy of the patch by a factor of $e^{3 N} \sim 10^{129}$. This is the massive entropy production. After "reheating" the patch contains an entropy of order $10^{143}$, and can easily contain the comoving volume that corresponds to our present Hubble radius, which is characterized by an entropy of "only" $10^{90}$.

It is clear that the smoothness problem has been solved. The kinematic requirement for producing density perturbations on astrophysically interesting scales has been satisfied; the mechanism that produces density perturbations, quantum fluctuations in the $\phi$ field, will be discussed later. What about the flatness problem? Suppose for definiteness that the curvature radius at the beginning of inflation is of order the Hubble radius (which corresponds to $\Omega$ just beginning to 
deviate from unity); at the end of inflation the curvature radius has grown by a factor of $e^{N}$, while the energy density has remained constant. This means that $\Omega_{\mathrm{end}}=1 /\left[1-\left(k / R^{2}\right) /(8 \pi G \rho / 3)\right] \simeq 1 \pm e^{-2 N}$ has been reset to a value exponentially close to unity. Using our fiducial numbers, at the end of inflation the curvature radius is order $10^{20} \mathrm{~cm}$; from then until today it grows by a factor of $\mathcal{M} / 3 \mathrm{~K} \sim 10^{27}$, reaching a present size of order $10^{47} \mathrm{~cm}$. This is enormous compared to the present Hubble radius and implies that $\Omega$ is still very close to unity today. The flatness problem has clearly been solved and a flat Universe predicted.

Consider the fate of monopoles - or any other "cosmic pollutant" in the preinflationary Universe. The number of monopoles within the patch $\left(=N_{M}\right)$ remains constant; however, the number per comoving volume, $n_{M} / s=N_{M} / S$, decreases by a factor of $e^{3 N} \sim 10^{129}$ due to the massive entropy production. Undesirables are diluted away! Of course, this also implies that the baryon number of the Universe, $n_{B} / s \sim 10^{-10}$, must be produced after inflation.

Finally, a simple exercise; what is the minimum amount of inflation needed to solve the smoothness problem? Start with a Hubble-sized patch at the beginning of inflation; it contains an entropy of $S_{\text {initial }} \sim H^{-3} T^{3} \sim\left(m_{\mathrm{Pl}} / \mathcal{M}\right)^{3}$. Assuming perfect conversion of vacuum energy to radiation, after inflation the entropy contained within the patch is $e^{3 N} S_{\text {initial }} \sim e^{3 N} m_{\mathrm{Pl}}^{3} / \mathcal{M}^{3}$. To solve the smoothness problems this must be greater than $10^{90}$, which implies

$$
N \gtrsim N_{\min }=56+\ln \left(\mathcal{M} / 10^{14} \mathrm{GeV}\right)
$$

Equivalently, one can express the size of the patch today relative to the present Hubble radius,

$$
d_{\text {patch }}=\exp \left(N-N_{\min }\right) H_{0}^{-1} .
$$

What about the flatness problem? It is simple to show the present value of $\Omega$ is related to that at the beginning of inflation and the size of the patch today:

$$
\left|\Omega_{0}-1\right|=\left(\frac{H_{0}^{-1}}{d_{\text {patch }}}\right)^{2}\left|\Omega_{\text {preinflation }}-1\right| .
$$

Remarkably enough, the amount of inflation required to solve the flatness and smoothness problems is the same. Put another way, if one comfortably solves the smoothness problem, $\Omega_{0}$ is necessarily very, very close to unity. This means that a flat Universe is an unequivocal prediction of inflation.

\subsection{Current Status of Inflationary Models}

\subsubsection{Types of inflation}

In this very brief overview I divide models of inflation into three broad classes: old, slow rollover, and first-order (or extended). By old inflation I mean Guth's original 
model, which I forgot to mention was a nonstarter! Let me explain; once trapped in the false vacuum, the Higgs field must quantum-mechanically tunnel to the true vacuum; in order to ensure a sufficient amount of inflation, this transition must not occur until 60 or so Hubble times after inflation has begun. As we shall see this is essentially impossible to arrange.

The decay of the false vacuum is well understood [38]: It proceeds via the nucleation of bubbles of true vacuum that expand outward at the speed of light. For a given potential the bubble nucleation rate (per unit volume) $\Gamma$ is straightforward to calculate [38]. Roughly speaking, bubbles convert all of space into the true vacuum when $\Gamma / H^{4}$, the number of bubbles nucleated in a Hubble volume in a Hubble time exceeds order unity; since each bubble nucleated during a Hubble time liberates about a Hubble volume, $\Gamma / H^{4} \sim 1$ ensures that all of space is converted to true-vacuum in a Hubble time (before the expansion "creates" more false vacuum). The false-vacuum energy is converted into "heat" by the collision of vacuum bubbles [39].

The recipe for successful old inflation is for $\Gamma / H^{4}$ to remain less than unity for 60 or so Hubble times and then increase to greater than unity. Unfortunately, shortly after inflation begins $\Gamma$, like the expansion rate becomes constant, as the temperature of the Universe rapidly approaches zero and become irrelevant. This is the fundamental problem with old inflation; $\Gamma / H^{4}$ is constant. The Universe can either get hung up in the false vacuum and inflate, or make the transition to the true vacuum, not both!

Slow-rollover inflation solved this problem, but at a price. The fix, suggested independently by Linde [40, and Albrecht and Steinhardt [41, is for inflation to occur as the scalar field slowly rolls the potential. They proposed using very flat potentials with small or nonexistent barriers between the false and true vacuum states; the vacuum-driven expansion takes place as the scalar field slowly (timescale $\gtrsim 60 \mathrm{H}^{-1}$ ), but inevitably rolls toward the true-vacuum state. When the scalar field responsible for inflation (often called the inflaton) reaches the true minimum of its potential it oscillates about it, the large vacuum energy having been converted into coherent inflaton oscillations. These oscillations ultimately decay into light-particle states reheating the Universe. From the quantum view, these coherent field oscillations correspond to zero-momentum inflaton particles; the decay of the scalar-field oscillations corresponds to the decay of massive inflaton particles [42].

Slow rollover led to the first viable models of inflation. There was, however, a price: In all models of slow-rollover the inflaton field must be very weakly coupled (dimensionless self coupling of order $10^{-14}$ or so); as we shall see this is dictated by achieving density perturbations of size $10^{-5}$ or so. Because of this fact, the inflaton cannot be directly responsible for GUT symmetry breaking as loop corrections from the inflaton-gauge interaction would spoil the flatness of the potential. The decoupled nature of the scalar field responsible for inflation gave birth to its name. In the broadest sense, slow-rollover inflation refers to any model of inflation where the a scalar field is displaced from the minimum of its potential and slowly rolls to the minimum. The minimum can be away from the origin, as with a potential associated with spontaneous symmetry breaking (often referred to as "new inflation"), or at the origin, e.g., $V(\phi)=\lambda \phi^{4}$ or $V(\phi)=m^{2} \phi^{2} / 2$ (often referred to as chaotic inflation). 
The latest and perhaps most interesting development in inflationary models is first-order (or extended) inflation [43]. In many ways it combines the best features of old inflation - intimate connection to particle physics phenomenology - and slowrollover inflation - it works! As the name suggests, these models are associated with a first-order phase transition; how then do these models solve the Guth dilemmathe constancy of $\Gamma / H^{4}$ ? The first model of this type was due to La and Steinhardt 44]; their new twist was to use the Brans-Dicke theory of gravity rather than general relativity. In Brans-Dicke the gravitational constant $G_{\text {eff }}=\Phi^{-2}$, and evolves as the Brans-Dicke field $\Phi$ evolves. Because of this, for constant energy density the scale factor only increases as a power of time, $R(t) \propto t^{\omega+1 / 2}$ and $H$ decreases with time; here $\omega$ is the coefficient of the kinetic-energy term for $\Phi$. Thus, the efficiency of bubble nucleation $\Gamma / H^{4} \propto t^{4}$ increases during inflation; at early times it can be much less than unity (so that the Universe remains trapped in the false vacuum) and then exceeds unity triggering the end of inflation via the nucleation and percolation of bubbles of true vacuum.

Models based on variations of this idea have been proposed. For example, if the Higgs field couples to other fields which are evolving during inflation, then $\Gamma$ will vary during inflation, leading to the variation of $\Gamma / H^{4}$ [45. In first-order inflation models the Higgs field plays a relatively passive role, remaining trapped in the false vacuum during inflation; further, it need not be weakly coupled, nor is the shape of its potential particularly relevant.

By means of a conformal transformation extended inflation can be recast as slowrollover inflation with an exponential potential with $\ln \Phi$ field playing the role of the inflaton 46]. In first-order inflation models there is another problem one has to worry about: If $\Gamma / H^{4}$ does not change rapidly enough, then too many bubbles will be nucleated long before the end of inflation; these bubbles eventually grow to astrophysical size and can have disastrous consequences (large anisotropies in the CBR, interference with primordial nucleosynthesis, and so on) [47]. To avoid "the big-bubble problem" in extended inflation $\omega$ must be less than about 20; that there be an upper limit to $\omega$ is not surprising since in the limit $\omega \rightarrow \infty$, Brans-Dicke goes to general relativity.

\subsubsection{Viable models}

There is no standard model of inflation; nor is there a model of inflation without some flaw. There are a number of "proof of existence" models, models that successful implement inflation, but are only beautiful in the eyes of their creators. Of course, this situation should be viewed in light of our general ignorance about physics at energy scales $\gg 10^{3} \mathrm{GeV}$ (most inflation models involve an energy scale of order $10^{14} \mathrm{GeV}$ ). Moreover, the same criticism - lack of a standard model - applies to baryogenesis, and applied to primordial nucleosynthesis until the early 1970's!

Slow-rollover. There are numerous viable models; I will mention but a representative few. There is an almost decade old model based upon an ordinary GUT due to 
Shafi and Vilenkin [48], and Pi [49]. This model has the virtue that the inflaton field does more than cause inflation; it also breaks Peccei-Quinn symmetry and induces GUT symmetry breaking (by producing a negative mass-squared for the GUT Higgs field). After inflation the Universe reheats to a temperature of order $10^{7} \mathrm{GeV}$, and a scenario for baryogenesis is included. In short, it is a complete model.

In passing, let me mention a similar model just proposed by Knox and myself [50]. The new twist is that the scale of inflation can be as small as the electroweak scale(!), and the inflaton field can be used to induce electroweak-symmetry breaking and other low-energy phenomena (e.g., righthanded neutrino masses). In principle, this model can be tested in laboratory experiments. Of course, this model is only viable provided one believes that the baryon asymmetry of the Universe can be produced at the weak scale or below.

There are many supersymmetric implementations of slow-rollover inflation [51]; a particularly elegant one is that of Holman, Ramond, and Ross [52]. The superpotential for their inflaton is very simple, $W(\phi)=\left(\Delta^{2} / M\right)(\phi-M)^{2}$; here $M=m_{\mathrm{Pl}} / \sqrt{8 \pi}$ and $\Delta$ is the GUT scale. In this model, the self coupling of the inflaton in its scalar potential is given by the fourth power of the ratio of the GUT to Planck scales, $(\Delta / M)^{4}$, and the canonical small number arises because of the discrepancy between the GUT and Planck scales. The reheat temperature in this model is order $10^{6} \mathrm{GeV}$, and the details of baryogenesis are spelled out.

There is a model called (by the authors) "natural inflation" [53]. The primary purpose of this model is to address the small self-coupling of the inflaton. To wit, the inflaton is a pseudo Nambu-Goldstone boson akin to the axion; a Nambu-Goldstone boson has an absolutely flat potential, i.e. is massless, and becomes a pseudo NambuGoldstone boson due to explicit symmetry breaking. The potential, $V(\phi)=\Lambda^{4}[1+$ $\cos (\phi / f)]$, has two energy scales: $f \sim m_{\mathrm{Pl}}$, the scale of the spontaneous symmetry breaking and $\Lambda \sim 10^{-5} f$, the scale of explicit symmetry breaking (GUT scale?). (In the axion analogy, $\Lambda=\Lambda_{\mathrm{QCD}} \simeq 200 \mathrm{MeV}$ and $f$ is the PQ symmetry-breaking scale.) Some superstring adherents have taken interest in this model as superstring theories often have pseudo Nambu-Goldstone bosons with Planck-scale symmetry breaking.

There is a broad class of slow-rollover models referred to as chaotic inflation; they illustrate the simplicity of inflation and were pioneered by Linde [54. The potentials for these models are not of the symmetry breaking variety as the minima are at $\phi=0$; e.g., $V(\phi)=\lambda \phi^{4}$ or $V(\phi)=m^{2} \phi^{2} / 2$. In chaotic inflation, the inflaton field begins far from the origin, $\phi \gtrsim 5 m_{\mathrm{Pl}}$ - the further the better. As with all slow-rollover models, there is a small, dimensionless number: $\lambda \sim 10^{-14}$ or $m^{2} \simeq 10^{-12} m_{\mathrm{Pl}}^{2}$. No attempt is made to connect these models with particle physics.

There are models where the inflaton field is not actually a scalar field; e.g., where it is related to the size of the compactified dimensions in models with extra dimensions [55], or is related to the scalar curvature $\mathcal{R}$ in higher derivative theories of gravity) 56 .

The common undesirable feature of all slow-rollover models is a small, dimensionless number of order $10^{-14}$, typically the self coupling of the inflaton; as we shall discuss, this small number is necessary to guarantee density perturbations of the ap- 
propriate size. To ensure the stability of the flatness of the potential against quantum (radiative) corrections the inflaton must be weakly coupled to the "rest of the world," and in this since, all the models mentioned are natural. However, weak coupling works at cross purposes with reheating and baryogenesis. Slow-rollover models liberate only a tiny fraction of the false-vacuum energy to radiation and have a relatively low reheat temperatures, which is problematic for baryogenesis as it must proceed after inflation. The second problem lies in the name "inflaton;" because the field responsible for inflation is so weakly coupled, without heroic efforts it is difficult to make it an integral part of a more encompassing particle physics theory.

First-order. These models have the potential (no pun intended) to incorporate the best aspects of both slow-rollover and old inflation. Inflation is again intimately connected to a cosmological phase transition at a scale of order the GUT scale and no special flatness is required of the Higgs potential. Moreover, reheating proceeds via vacuum-bubble collisions which guarantees good reheating and a unique signature of first-order inflation, a background of gravitational waves proceeded by bubble collisions, $\Omega_{\mathrm{GW}} \sim 10^{-8}$ at a frequency determined by the scale of inflation, $f_{\mathrm{GW}} \sim 10^{6} \mathrm{~Hz}\left(\mathcal{M} / 10^{12} \mathrm{GeV}\right)$ [57].

The simplest first-order inflation model is extended inflation. First the good news: Brans-Dicke gravity exhibits conformal (scale) invariance (the Planck scale is replaced by a field). Conformal invariance is "the Hallmark" of superstring theory, which has stimulated new interest in Brans-Dicke like theories. Now the bad news; in order to avoid "the big-bubble problem," the Brans-Dicke parameter $\omega$ must be less than about 20, while solar-system tests set a lower limit of about 500 [58. In its simplest form, extended inflation is not viable. Several variants have been put forth 43]; the simplest fix is to give the Brans-Dicke field a mass [46]. (A mass for the Brans-Dicke field anchors at the right value and makes the immune to solar-system tests.) Any mass less than about $10^{9} \mathrm{GeV}$ and greater than a tiny fraction of an $\mathrm{eV}$ will do. Moreover, this simple fix involves something that string theorists must do anyway: break conformal invariance (the world is not conformally invariant, it has a multitude of energy scales).

In sum, inflation provides a very attractive early Universe paradigm. Models of inflation are based upon well defined, albeit very speculative, physics at energy scales well below the Planck scale. At present there is no standard model, or even a particularly compelling model; there are, however, a variety of models that work. Given our general ignorance about physics at energy scales $\gg 10^{3} \mathrm{GeV}$, perhaps that should be enough for the time being. In any case, while elegance, simplicity, and mathematical beauty often provide guidance to the theorist, in the end, experiment and observation are the final arbiters. As I will discuss toward the end, observations involving structure formation are starting to do just that. 


\subsection{Initial Conditions: No-hair theorems}

Inflation is cosmologically attractive because it promises to account for our present nearly FRW space-time starting from very general initial conditions. Somewhat paradoxically, inflation is usually analyzed in the context of the isotropic and homogeneous FRW cosmology. I will now explain the apparent paradox and discuss to what extent inflation lessens the dependence of the present state of the Universe upon its initial state.

To begin consider the anisotropic but homogeneous (Bianchi) models; the mean expansion rate of the Universe can be written as

$$
H^{2} \equiv(\dot{\bar{R}} / \bar{R})^{2}=\frac{8 \pi G \rho}{3}+F(\dot{\bar{R}}, \bar{R}) ;
$$

where $\bar{R}$ is the mean scale factor and $\rho$ is the usual energy density and the function $F$ accounts for the additional terms that arise due to anisotropy. In general, the function $F$ decreases at least as rapidly as $1 / \bar{R}^{2}$, that is, as rapidly as the spatial curvature term in the FRW cosmology or faster. The false-vacuum energy density appears in the energy density term and is of course constant. Provided that $F$ is positive, the Universe will eventually become vacuum-energy dominated; once it does, the $F(\dot{R}, \bar{R})$ term will quickly decrease and become insignificant and the space time becomes isotropic. This justifies the usual FRW analysis of inflation.

Not all anisotropic space-times will inflate; if $F$ is sufficiently large and negative it will prevent inflation; the simplest noninflating model is a very positively curved FRW model that recollapses before it can inflate. The strongly positively curved models preclude a true cosmological no-hair theorem; however, it has been shown that all spatially homogeneous, but anisotropic models eventually inflate, except for the very positively curved models [60]. And further, it has been shown that "smooth regions" of inhomogeneous models of sufficient size and that are negatively curved will inflate [61, 62]. While not all spacetimes will inflate, the class of spacetimes that do is not special, but very generic 62. Thus, inflation does indeed lessen the dependence of the present state of the Universe on its initial state.

Does inflation render a generic space-time isotropic and homogeneous forever? The answer is clearly no; the most one can expect in an inhomogeneous space-time is that negatively curved regions inflate. Further, once inflation is over, inhomogeneity and anisotropy will "grow back." Consider spatial curvature; if the Universe was not flat before inflation it will not be flat after inflation. However, inflation exponentially postpones the epoch when spatial curvature becomes important because the value of $\Omega$ after inflation becomes exponential close to unity. Likewise, in the exponential distant future our Hubble volume will become larger than the generic smooth patch

\footnotetext{
${ }^{5}$ There is one worry; namely that the inflaton field will evolve to the minimum of its potential before the vacuum-dominated phase begins. In general, this does not occur as anisotropy increases the expansion rate, and thus the friction term in the equation of motion for the inflaton; see 59.
} 
created by inflation and we will in principle we able to see the inhomogeneity beyond our inflationary patch 63.

Finally, there are the initial data for the scalar field responsible for inflation itself. In first-order inflation, as in old inflation, this is a dynamical issue: the initial value of the scalar field is determined by thermal considerations. However, in slow-rollover inflation the story is very different; the initial value of the inflaton field (and its spatial and temporal derivatives) are not so determined, and at the classical level must be considered to be initial data. While this has become a subject unto itself, some very general statements can be made. First, the inflaton field must be smooth on a scale comparable to the Hubble radius, otherwise the energy density associated with spatial gradients will dominate over the vacuum energy preventing inflation. Second, the value of the scalar field must be small enough in models of "new inflation" or large enough in models of "chaotic inflation" so that it takes the field more than 60 Hubble times to roll to the bottom of the potential. Finally, the initial velocity of the inflaton (i.e., $\dot{\phi}$ ) must be small enough so that it does not rapidly speed to the bottom of the potential. For a given inflationary model, all of these considerations can be studied and quantitative statements made about the necessary initial data for the inflaton field 64]; further, attempts have been made using the wavefunction of the Universe to quantify the quantum expectation for the initial state of the inflaton field [65].

In the final analysis it cannot be said that all initial spacetimes undergo inflation and become isotropic and homogeneous for all time; further, the initial data for the inflaton itself must now be considered. The strongest statement that one can make is to say that inflation greatly lessens the dependence of the present state of the Universe upon its initial state. In my mind, that's no mean feat and inflation should be considered a great success.

\section{Inflation: The Fundamentals}

In this Section I discuss how to analyze an inflationary model, given the scalar potential. In two sections hence I will work through a number of examples. The focus will be on the metric perturbations - density fluctuations [66] and gravity waves [67]that arise due to quantum fluctuations, and the CBR temperature anisotropies that result from them. ๆ Perturbations on all astrophysically interesting scales, say $1 \mathrm{Mpc}$ to $10^{4} \mathrm{Mpc}$, are produced during an interval of about 8 e-folds around 50 e-folds before the end of inflation, when these scales crossed outside the horizon during inflation. I will show how the density perturbations and gravity waves can be related to three features of the inflationary potential: its value $V_{50}$, its steepness $x_{50} \equiv\left(m_{\mathrm{Pl}} V^{\prime} / V\right)_{50}$, and the change in its steepness $x_{50}^{\prime}$, evaluated in the region of the potential where the

\footnotetext{
${ }^{6}$ Isocurvature perturbations can arise due to quantum fluctuations in other massless fields, e.g., the axion field, if it exists [69].
} 
scalar field was about 50 e-folds before the end of inflation. In principle, cosmological observations, most importantly CBR anisotropy, can be used to determine the characteristics of the density perturbations and gravitational waves and thereby $V_{50}, x_{50}$, and $x_{50}^{\prime}$.

All viable models of inflation are of the slow-rollover variety, or can be recast as such 68. In slow-rollover inflation a scalar field that is initially displaced from the minimum of its potential rolls slowly to that minimum, and as it does the cosmicscale factor grows very rapidly. Once the scalar field reaches the minimum of the potential it oscillates about it, so that the large potential energy has been converted into coherent scalar-field oscillations, corresponding to a condensate of nonrelativistic scalar particles. The eventual decay of these particles into lighter particle states and their subsequent thermalization lead to the reheating of the Universe to a temperature $T_{\mathrm{RH}} \simeq \sqrt{\Gamma m_{\mathrm{Pl}}}$, where $\Gamma$ is the decay width of the scalar particle 42, 68. Here, I will focus on the classical evolution of the inflaton field during the slow-roll phase and the small quantum fluctuations in the inflaton field which give rise to density perturbations and those in the metric which give rise to gravity waves.

To begin, let us assume that the scalar field driving inflation is minimally coupled so that its stress-energy tensor takes the canonical form,

$$
T_{\mu \nu}=\partial_{\mu} \phi \partial_{\nu} \phi-\mathcal{L} g_{\mu \nu}
$$

where the Lagrangian density of the scalar field $\mathcal{L}=\frac{1}{2} \partial_{\mu} \phi \partial^{\mu} \phi-V(\phi)$. If we make the usual assumption that the scalar field $\phi$ is spatially homogeneous, or at least so over a Hubble radius, the stress-energy tensor takes the perfect-fluid form with energy density, $\rho=\frac{1}{2} \dot{\phi}^{2}+V(\phi)$, and isotropic pressure, $p=\frac{1}{2} \dot{\phi}^{2}-V(\phi)$. The classical equations of motion for $\phi$ can be obtained from the first law of thermodynamics, $d\left(R^{3} \rho\right)=-p d R^{3}$, or by taking the four-divergence of $T^{\mu \nu}$ :

$$
\ddot{\phi}+3 H \dot{\phi}+V^{\prime}(\phi)=0
$$

the $\Gamma \dot{\phi}$ term responsible for reheating has been omitted since we shall only be interested in the slow-rollover phase. In addition, there is the Friedmann equation, which governs the expansion of the Universe,

$$
H^{2}=\frac{8 \pi}{3 m_{\mathrm{Pl}}^{2}}\left(V(\phi)+\frac{1}{2} \dot{\phi}^{2}\right) \simeq \frac{8 \pi V(\phi)}{3 m_{\mathrm{Pl}}^{2}}
$$

where we assume that the contribution of all other forms of energy density, e.g., radiation and kinetic energy of the scalar field, and the curvature term $\left(k / R^{2}\right)$ are negligible. The justification for discussing inflation in the context of a flat FRW model with a homogeneous scalar field driving inflation were discussed earlier (and at greater length in Ref. [70]); including the $\phi$ kinetic term increases the righthand side of Eq. (31) by a factor of $\left(1+x^{2} / 48 \pi\right)$, a small correction for viable models.

In the next Section I will be more precise about the amplitude of density perturbations and gravitational waves; for now, let me briefly discuss how these perturbations 
arise and give their characteristic amplitudes. The metric perturbations produced in inflationary models are very nearly "scale invariant," a particularly simple spectrum which was first discussed by Harrison and Zel'dovich [71], and arise due to quantum fluctuations. In deSitter space all massless scalar fields experience quantum fluctuations of amplitude $H / 2 \pi$. The graviton is massless and can be described by two massless scalar fields, $h_{+, \times}=\sqrt{16 \pi G} \phi_{+, \times}(+$and $\times$are the two polarization states $)$. The inflaton by virtue of its flat potential is for all practical purposes massless.

Fluctuations in the inflaton field lead to density fluctuations because of its scalar potential, $\delta \rho \sim H V^{\prime}$; as a given mode crosses outside the horizon, the density perturbation on that scale becomes a classical metric perturbation. While outside the horizon, the description of the evolution of a density perturbation is beset with subtleties associated with the gauge freedom in general relativity; there is, however, a simple gauge-invariant quantity, $\zeta \simeq \delta \rho /(\rho+p)$, which remains constant outside the horizon. By equating the value of $\zeta$ at postinflation horizon crossing with its value as the scale crosses outside the horizon it follows that $(\delta \rho / \rho)_{\mathrm{HOR}} \sim H V^{\prime} / \dot{\phi}^{2}$ (note: $\left.\rho+p=\dot{\phi}^{2}\right)$; see Fig. 7 .

The evolution of a gravity-wave perturbation is even simpler; it obeys the massless Klein-Gordon equation

$$
\ddot{h}_{k}^{i}+3 H \dot{h}_{k}^{i}+k^{2} h_{k}^{i} / R^{2}=0 ;
$$

where $k$ is the wavenumber of the mode and $i=+, \times$. For superhorizon sized modes, $k \lesssim R H$, the solution is simple: $h_{k}^{i}=$ const. Like their density perturbation counterparts, gravity-wave perturbations become classical metric perturbations as they cross outside the horizon; they are characterized by an amplitude $h_{k}^{i} \simeq \sqrt{16 \pi G}(H / 2 \pi) \sim H / m_{\mathrm{Pl}}$. At postinflation horizon crossing their amplitude is unchanged.

Finally, let me write the horizon-crossing amplitudes of the scalar and tensor metric perturbations in terms of the inflationary potential,

$$
\begin{aligned}
(\delta \rho / \rho)_{\mathrm{HOR}, \lambda} & =c_{S}\left(\frac{V^{3 / 2}}{m_{\mathrm{Pl}}^{3} V^{\prime}}\right)_{1} ; \\
h_{\mathrm{HOR}, \lambda} & =c_{T}\left(\frac{V^{1 / 2}}{m_{\mathrm{Pl}}^{2}}\right)_{1} ;
\end{aligned}
$$

where $(\delta \rho / \rho)_{\mathrm{HOR}, \lambda}$ is the amplitude of the density perturbation on the scale $\lambda$ when it crosses the Hubble radius during the post-inflation epoch, $h_{\mathrm{HOR}, \lambda}$ is the dimensionless amplitude of the gravitational wave perturbation on the scale $\lambda$ when it crosses the Hubble radius, and $c_{S}, c_{T}$ are numerical constants of order unity. Subscript 1 indicates that the quantity involving the scalar potential is to be evaluated when the scale in question crossed outside the horizon during the inflationary era. The metric perturbations produced by inflation are characterized by almost scale-invariant horizon-crossing amplitudes; the slight deviations from scale invariance result from the variation of $V$ and $V^{\prime}$ during inflation which enter through the dependence upon $t_{1}$. [In Eq. (33) I got ahead of myself and used the slow-roll approximation (see 
below) to rewrite the expression, $(\delta \rho / \rho)_{\mathrm{HOR}, \lambda} \simeq H V^{\prime} / \dot{\phi}$, in terms of the potential only.]

Eqs. (30-33) are the fundamental equations that govern inflation and the production of metric perturbations. It proves very useful to recast these equations using the scalar field as the independent variable; we then express the scalar and tensor perturbations in terms of the value of the potential, its steepness, and the rate of change of its steepness when the interesting scales crossed outside the Hubble radius during inflation, about 50 e-folds in scale factor before the end of inflation, defined by

$$
V_{50} \equiv V\left(\phi_{50}\right) ; \quad x_{50} \equiv \frac{m_{\mathrm{Pl}} V^{\prime}\left(\phi_{50}\right)}{V\left(\phi_{50}\right)} ; \quad x_{50}^{\prime}=\frac{m_{\mathrm{Pl}} V^{\prime \prime}\left(\phi_{50}\right)}{V\left(\phi_{50}\right)}-\frac{m_{\mathrm{Pl}}\left[V^{\prime}\left(\phi_{50}\right)\right]^{2}}{V^{2}\left(\phi_{50}\right)}
$$

To evaluate these three quantities 50 e-folds before the end of inflation we must find the value of the scalar field at this time. During the inflationary phase the $\ddot{\phi}$ term is negligible (the motion of $\phi$ is friction dominated), and Eq. (30) becomes

$$
\dot{\phi} \simeq \frac{-V^{\prime}(\phi)}{3 H}
$$

this is known as the slow-roll approximation [72. While the slow-roll approximation is almost universally applicable, there are models where the slow-roll approximation cannot be used; e.g., a potential where during the crucial 8 e-folds the scalar field rolls uphill, "powered" by the velocity it had when it hit the incline.

The conditions that must be satisfied in order that $\ddot{\phi}$ be negligible are:

$$
\begin{aligned}
\left|V^{\prime \prime}\right| & <9 H^{2} \simeq 24 \pi V / m_{\mathrm{Pl}}^{2} ; \\
|x| \equiv\left|V^{\prime} m_{\mathrm{Pl}} / V\right| & <\sqrt{48 \pi} .
\end{aligned}
$$

The end of the slow roll occurs when either or both of these inequalities are saturated, at a value of $\phi$ denoted by $\phi_{\text {end }}$. Since $H \equiv \dot{R} / R$, or $H d t=d \ln R$, it follows that

$$
d \ln R=\frac{8 \pi}{m_{\mathrm{Pl}}^{2}} \frac{V(\phi) d \phi}{-V^{\prime}(\phi)}=-\frac{8 \pi d \phi}{m_{\mathrm{Pl}} x} .
$$

Now express the cosmic-scale factor in terms of is value at the end of inflation, $R_{\text {end }}$, and the number of e-foldings before the end of inflation, $N(\phi)$,

$$
R=\exp [-N(\phi)] R_{\mathrm{end}}
$$

The quantity $N(\phi)$ is a time-like variable whose value at the end of inflation is zero and whose evolution is governed by

$$
\frac{d N}{d \phi}=\frac{8 \pi}{m_{\mathrm{Pl}} x}
$$


Using Eq. (39) we can compute the value of the scalar field 50 e-folds before the end of inflation $\left(\equiv \phi_{50}\right)$; the values of $V_{50}, x_{50}$, and $x_{50}^{\prime}$ follow directly.

As $\phi$ rolls down its potential during inflation its energy density decreases, and so the growth in the scale factor is not exponential. By using the fact that the stressenergy of the scalar field takes the perfect-fluid form, we can solve for evolution of the cosmic-scale factor. Recall, for the equation of state $p=\gamma \rho$, the scale factor grows as $R \propto t^{q}$, where $q=2 / 3(1+\gamma)$. Here,

$$
\begin{aligned}
\gamma & =\frac{\frac{1}{2} \dot{\phi}^{2}-V}{\frac{1}{2} \dot{\phi}^{2}+V}=\frac{x^{2}-48 \pi}{x^{2}+48 \pi} \\
q & =\frac{1}{3}+\frac{16 \pi}{x^{2}}
\end{aligned}
$$

Since the steepness of the potential can change during inflation, $\gamma$ is not in general constant; the power-law index $q$ is more precisely the logarithmic rate of the change of the logarithm of the scale factor, $q=d \ln R / d \ln t$.

When the steepness parameter is small, corresponding to a very flat potential, $\gamma$ is close to -1 and the scale factor grows as a very large power of time. To solve the horizon problem the expansion must be "superluminal" $(\ddot{R}>0)$, corresponding to $q>1$, which requires that $x^{2}<24 \pi$. Since $\frac{1}{2} \dot{\phi}^{2} / V=x^{2} / 48 \pi$, this implies that $\frac{1}{2} \dot{\phi}^{2} / V(\phi)<\frac{1}{2}$, justifying neglect of the scalar-field kinetic energy in computing the expansion rate for all but the steepest potentials. (In fact there are much stronger constraints; the COBE DMR data imply that $n \gtrsim 0.5$, which restricts $x_{50}^{2} \lesssim 4 \pi$, $\frac{1}{2} \dot{\phi}^{2} / V \lesssim \frac{1}{12}$, and $q \gtrsim 4$.)

Next, let us relate the size of a given scale to when that scale crosses outside the Hubble radius during inflation, specified by $N_{1}(\lambda)$, the number of e-folds before the end of inflation. The physical size of a perturbation is related to its comoving size, $\lambda_{\text {phys }}=R \lambda$; with the usual convention, $R_{\text {today }}=1$, the comoving size is the physical size today. When the scale $\lambda$ crosses outside the Hubble radius $R_{1} \lambda=H_{1}^{-1}$. We then assume that: (1) at the end of inflation the energy density is $\mathcal{M}^{4} \simeq V\left(\phi_{\text {end }}\right)$; (2) inflation is followed by a period where the energy density of the Universe is dominated by coherent scalar-field oscillations which decrease as $R^{-3}$; and (3) when value of the scale factor is $R_{\mathrm{RH}}$ the Universe reheats to a temperature $T_{\mathrm{RH}} \simeq \sqrt{m_{\mathrm{Pl}} \Gamma}$ and expands adiabatically thereafter. The "matching equation" that relates $\lambda$ and $N_{1}(\lambda)$ is:

$$
\lambda=\frac{R_{\text {today }}}{R_{1}} H_{1}^{-1}=\frac{R_{\text {today }}}{R_{\mathrm{RH}}} \frac{R_{\mathrm{RH}}}{R_{\text {end }}} \frac{R_{\text {end }}}{R_{1}} H_{1}^{-1} .
$$

Adiabatic expansion since reheating implies $R_{\text {today }} / R_{\mathrm{RH}} \simeq T_{\mathrm{RH}} / 2.73 \mathrm{~K}$; and the decay of the coherent scalar-field oscillations implies $\left(R_{\mathrm{RH}} / R_{\text {end }}\right)^{3}=\left(\mathcal{M} / T_{\mathrm{RH}}\right)^{4}$. If we define $\bar{q}=\ln \left(R_{\text {end }} / R_{1}\right) / \ln \left(t_{\text {end }} / t_{1}\right)$, the mean power-law index, it follows that $\left(R_{\text {end }} / R_{1}\right) H_{1}^{-1}=\exp \left[N_{1}(\bar{q}-1) / \bar{q}\right] H_{\text {end }}^{-1}$, and Eq. (42) becomes

$$
N_{1}(\lambda)=\frac{\bar{q}}{\bar{q}-1}\left[48+\ln \lambda_{\mathrm{Mpc}}+\frac{2}{3} \ln \left(\mathcal{M} / 10^{14} \mathrm{GeV}\right)+\frac{1}{3} \ln \left(T_{\mathrm{RH}} / 10^{14} \mathrm{GeV}\right)\right]
$$


In the case of perfect reheating, which probably only applies to first-order inflation, $T_{\mathrm{RH}} \simeq \mathcal{M}$.

The scales of astrophysical interest today range roughly from that of galaxy size, $\lambda \sim$ Mpc, to the present Hubble scale, $H_{0}^{-1} \sim 10^{4} \mathrm{Mpc}$; up to the logarithmic corrections these scales crossed outside the horizon between about $N_{1}(\lambda) \sim 48$ and $N_{1}(\lambda) \simeq 56$ e-folds before the end of inflation. That is, the interval of inflation that determines its all observable consequences covers only about 8 e-folds.

Except in the case of strict power-law inflation $q$ varies during inflation; this means that the $\left(R_{\text {end }} / R_{1}\right) H_{1}^{-1}$ factor in Eq. (42) cannot be written in closed form. Taking account of this, the matching equation becomes a differential equation,

$$
\frac{d \ln \lambda_{\mathrm{Mpc}}}{d N_{1}}=\frac{q\left(N_{1}\right)-1}{q\left(N_{1}\right)} ;
$$

subject to the "boundary condition:"

$$
\ln \lambda_{\mathrm{Mpc}}=-48-\frac{4}{3} \ln \left(\mathcal{M} / 10^{14} \mathrm{GeV}\right)+\frac{1}{3} \ln \left(T_{\mathrm{RH}} / 10^{14} \mathrm{GeV}\right)
$$

for $N_{1}=0$, the matching relation for the mode that crossed outside the Hubble radius at the end of inflation. Equation (44) allows one to obtain the precise expression for when a given scale crossed outside the Hubble radius during inflation. To actually solve this equation, one would need to supplement it with the expressions $d N / d \phi=$ $8 \pi / m_{\mathrm{Pl}} x$ and $q=16 \pi / x^{2}$. For our purposes we need only know: (1) The scales of astrophysical interest correspond to $N_{1} \sim$ " $50 \pm 4$," where for definiteness we will throughout take this to be an equality sign. (2) The expansion of Eq. (44) about $N_{1}=50$,

$$
\Delta N_{1}(\lambda)=\left(\frac{q_{50}-1}{q_{50}}\right) \Delta \ln \lambda_{\mathrm{Mpc}}
$$

which, with the aid of Eq. (39), implies that

$$
\Delta \phi=\left(\frac{q_{50}-1}{q_{50}}\right) \frac{x_{50}}{8 \pi} \Delta \lambda_{\mathrm{Mpc}} .
$$

We are now ready to express the perturbations in terms of $V_{50}, x_{50}$, and $x_{50}^{\prime}$. First, we must solve for the value of $\phi, 50$ e-folds before the end of inflation. To do so we use Eq. (39),

$$
N\left(\phi_{50}\right)=50=\frac{8 \pi}{m_{\mathrm{Pl}}{ }^{2}} \int_{\phi_{\mathrm{end}}}^{\phi_{50}} \frac{V d \phi}{V^{\prime}} .
$$

Next, with the help of Eq. (46) we expand the potential $V$ and its steepness $x$ about $\phi_{50}$ :

$$
\begin{gathered}
V \simeq V_{50}+V_{50}^{\prime}\left(\phi-\phi_{50}\right)=V_{50}\left[1+\frac{x_{50}^{2}}{8 \pi}\left(\frac{q_{50}}{q_{50}-1}\right) \Delta \ln \lambda_{\mathrm{Mpc}}\right] ; \\
x \simeq x_{50}+x_{50}^{\prime}\left(\phi-\phi_{50}\right)=x_{50}\left[1+\frac{m_{\mathrm{Pl}} x_{50}^{\prime}}{8 \pi}\left(\frac{q_{50}}{q_{50}-1}\right) \Delta \ln \lambda_{\mathrm{Mpc}}\right] ;
\end{gathered}
$$


of course these expansions only make sense for potentials that are smooth. We note that additional terms in either expansion are $\mathcal{O}\left(\alpha_{i}^{2}\right)$ and beyond the accuracy we are seeking.

Now recall the equations for the amplitude of the scalar and tensor perturbations,

$$
\begin{aligned}
(\delta \rho / \rho)_{\mathrm{HOR}, \lambda} & =c_{S}\left(\frac{V^{1 / 2}}{m_{\mathrm{Pl}}^{2} x}\right)_{1} ; \\
h_{\mathrm{HOR}, \lambda} & =c_{T}\left(\frac{V^{1 / 2}}{m_{\mathrm{Pl}}^{2}}\right)_{1} ;
\end{aligned}
$$

where subscript 1 means that the quantities are to be evaluated where the scale $\lambda$ crossed outside the Hubble radius, $N_{1}(\lambda)$ e-folds before the end of inflation. The origin of any deviation from scale invariance is clear: For tensor perturbations it arises due to the variation of the potential; and for scalar perturbations it arises due to the variation of both the potential and its steepness.

Using Eqs. (45-50) it is now simple to calculate the power-law exponents $\alpha_{S}$ and $\alpha_{T}$ that quantify the deviations from scale invariance,

$$
\begin{aligned}
\alpha_{T} & =\frac{x_{50}^{2}}{16 \pi} \frac{q_{50}}{q_{50}-1} \simeq \frac{x_{50}^{2}}{16 \pi} \\
\alpha_{S} & =\alpha_{T}-\frac{m_{\mathrm{Pl}} x_{50}^{\prime}}{8 \pi} \frac{q_{50}}{q_{50}-1} \simeq \frac{x_{50}^{2}}{16 \pi}-\frac{m_{\mathrm{Pl}} x_{50}^{\prime}}{8 \pi}
\end{aligned}
$$

where

$$
\begin{aligned}
q_{50} & =\frac{1}{3}+\frac{16 \pi}{x_{50}^{2}} \simeq \frac{16 \pi}{x_{50}^{2}} \\
h_{\mathrm{HOR}, \lambda} & =c_{T}\left(\frac{V_{50}^{1 / 2}}{m_{\mathrm{Pl}}^{2}}\right) \lambda_{\mathrm{Mpc}}^{\alpha_{T}} \\
(\delta \rho / \rho)_{\mathrm{HOR}, \lambda} & =c_{S}\left(\frac{V_{50}^{1 / 2}}{x_{50} m_{\mathrm{Pl}}^{2}}\right) \lambda_{\mathrm{Mpc}}^{\alpha_{S}} .
\end{aligned}
$$

The spectral indices $\alpha_{i}$ are defined as, $\alpha_{S}=\left[d \ln (\delta \rho / \rho)_{\mathrm{HOR}, \lambda} / d \ln \lambda_{\mathrm{Mpc}}\right]_{50}$ and $\alpha_{T}=$ $\left[d \ln h_{\mathrm{HOR}, \lambda} / d \ln \lambda_{\mathrm{Mpc}}\right]_{50}$, and in general vary slowly with scale. Note too that the deviations from scale invariance, quantified by $\alpha_{S}$ and $\alpha_{T}$, are of the order of $x_{50}^{2}$, $m_{\mathrm{Pl}} x_{50}^{\prime}$. In the expressions above we retained only lowest-order terms in $\mathcal{O}\left(\alpha_{i}\right)$. The next-order contributions to the spectral indices are $\mathcal{O}\left(\alpha_{i}^{2}\right)$; those to the amplitudes are $\mathcal{O}\left(\alpha_{i}\right)$ and are given two sections hence. The justification for truncating the expansion at lowest order is that the deviations from scale invariance are expected to be small — and are required by astrophysically data to be small.

As I discuss in more detail two sections hence, the more intuitive power-law indices $\alpha_{S}, \alpha_{T}$ are related to the indices that are usually used to describe the power spectra 
of scalar and tensor perturbations, $P_{S}(k)=\left|\delta_{k}\right|^{2}=A k^{n}$ and $P_{T}(k)=\left|h_{k}\right|^{2}=A_{T} k^{n_{T}}$,

$$
\begin{aligned}
n & =1-2 \alpha_{S}=1-\frac{x_{50}^{2}}{8 \pi}+\frac{m_{\mathrm{Pl}} x_{50}^{\prime}}{4 \pi} ; \\
n_{T} & =-2 \alpha_{T}=-\frac{x_{50}^{2}}{8 \pi} .
\end{aligned}
$$

CBR temperature fluctuations on large-angular scales $\left(\theta \gtrsim 1^{\circ}\right)$ due to metric perturbations arise through the Sachs-Wolfe effect; very roughly, the temperature fluctuation on a given angular scale $\theta$ is related to the metric fluctuation on the length scale that subtends that angle at last scattering, $\lambda \sim 100 h^{-1} \mathrm{Mpc}(\theta / \mathrm{deg})$,

$$
\begin{aligned}
& \left(\frac{\delta T}{T}\right)_{\theta} \sim\left(\frac{\delta \rho}{\rho}\right)_{\mathrm{HOR}, \lambda} ; \\
& \left(\frac{\delta T}{T}\right)_{\theta} \sim h_{\mathrm{HOR}, \lambda} ;
\end{aligned}
$$

where the scalar and tensor contributions to the CBR temperature anisotropy on a given scale add in quadrature. Let me be more specific about the amplitude of the quadrupole CBR anisotropy. For small $\alpha_{S}, \alpha_{T}$ the contributions of each to the quadrupole CBR temperature anisotropy:

$$
\begin{aligned}
& \left(\frac{\Delta T}{T_{0}}\right)_{Q-S}^{2} \approx \frac{32 \pi}{45} \frac{V_{50}}{m_{\mathrm{Pl}}^{4} x_{50}^{2}} \\
& \left(\frac{\Delta T}{T_{0}}\right)_{Q-T}^{2} \approx 0.61 \frac{V_{50}}{m_{\mathrm{Pl}}^{4}} \\
& \frac{T}{S} \equiv \frac{\left(\Delta T / T_{0}\right)_{Q-T}^{2}}{\left(\Delta T / T_{0}\right)_{Q-S}^{2}} \approx 0.28 x_{50}^{2}
\end{aligned}
$$

where expressions have been evaluated to lowest order in $x_{50}^{2}$ and $m_{\mathrm{Pl}} x_{50}^{\prime}$. These quantities represent the ensemble averages of the scalar and tensor contributions to the quadrupole temperature anisotropy, which in terms of the spherical-harmonic expansion of the CBR temperature anisotropy on the sky are given by $5\left\langle\left|a_{2 m}\right|^{2}\right\rangle / 4 \pi$. Further, the scalar and tensor contributions to the measured quadrupole anisotropy add in quadrature, and are subject to "cosmic variance." (Cosmic variance refers to the dispersion in the values measured by different observers in the Universe.)

Before going on, some general remarks [78]. The steepness parameter $x_{50}^{2}$ must be less than about $24 \pi$ to ensure superluminal expansion. For "steep" potentials, the expansion rate is "slow," i.e., $q_{50}$ closer to unity, the gravity-wave contribution to the quadrupole CBR temperature anisotropy becomes comparable to, or greater than, that of density perturbations, and both scalar and tensor perturbations exhibit significant deviations from scale invariance. For "flat" potentials, i.e., small $x_{50}$, the 
expansion rate is "fast," i.e., $q_{50} \gg 1$, the gravity-wave contribution to the quadrupole CBR temperature anisotropy is much smaller than that of density perturbations, and the tensor perturbations are scale invariant. Unless the steepness of the potential changes rapidly, i.e., large $x_{50}^{\prime}$, the scalar perturbations are also scale invariant.

\subsection{Metric perturbations and CBR anisotropy}

I was purposefully vague when discussing the amplitudes of the scalar and tensor modes, except when specifying their contributions to the quadrupole CBR temperature anisotropy; in fact, the spectral indices $\alpha_{S}$ and $\alpha_{T}$, together with the scalar and tensor contributions to the CBR quadrupole serve to provide all the information necessary. Here I will fill in more details about the metric perturbations.

The scalar and tensor metric perturbations are expanded in harmonic functions, in the flat Universe predicted by inflation, plane waves,

$$
\begin{aligned}
h_{\mu \nu}(\mathbf{x}, t) & =\frac{1}{(2 \pi)^{3}} \int d^{3} k h_{\mathbf{k}}^{i}(t) \varepsilon_{\mu \nu}^{i} e^{-i \mathbf{k} \cdot \mathbf{x}} ; \\
\frac{\delta \rho(\mathbf{x}, t)}{\rho} & =\frac{1}{(2 \pi)^{3}} \int d^{3} k \delta_{\mathbf{k}}(t) e^{-i \mathbf{k} \cdot \mathbf{x}}
\end{aligned}
$$

where $h_{\mu \nu}=R^{-2} g_{\mu \nu}-\eta_{\mu \nu}, \varepsilon_{\mu \nu}^{i}$ is the polarization tensor for the gravity-wave modes, and $i=+, \times$ are the two polarization states. Everything of interest can be computed in terms of $h_{\mathbf{k}}^{i}$ and $\delta_{\mathbf{k}}$. For example, the rms mass fluctuation in a sphere of radius $r$ is obtained in terms of the window function for a sphere and the power spectrum $P_{S}(k) \equiv\left\langle\left|\delta_{\mathbf{k}}\right|^{2}\right\rangle$ (see below),

$$
\left\langle(\delta M / M)^{2}\right\rangle_{r}=\frac{9}{2 \pi^{2} r^{2}} \int_{0}^{\infty}\left[j_{1}(k r)\right]^{2} P_{S}(k) d k ;
$$

where $j_{1}(x)$ is the spherical Bessel function of first order. If $P_{S}(k)$ is a power law, it follows roughly that $(\delta M / M)^{2} \sim k^{3}\left|\delta_{\mathbf{k}}\right|^{2}$, evaluated on the scale $k=r^{-1}$. This is what I meant by $(\delta \rho / \rho)_{\text {HOR }, \lambda}$ : the rms mass fluctuation on the scale $\lambda$ when it crossed inside the horizon. Likewise, by $h_{\mathrm{HOR}, \lambda}$ I meant the $r m s$ strain on the scale $\lambda$ as it crossed inside the Hubble radius, $\left(h_{\mathrm{HOR}, \lambda}\right)^{2} \sim k^{3}\left|h_{\mathbf{k}}^{i}\right|^{2}$.

In the previous discussions I have chosen to specify the metric perturbations for the different Fourier modes when they crossed inside the horizon, rather than at a common time. I did so because scale invariance is made manifest, as the scale independence of the metric perturbations at post-inflation horizon crossing. Recall, in the case of scalar perturbations $(\delta \rho / \rho)_{\mathrm{HOR}}$ is up to a numerical factor the fluctuation in the Newtonian potential, and, by specifying the scalar perturbations at horizon crossing, we avoid the discussion of scalar perturbations on superhorizon scales, which is beset by the subtleties associated with the gauge noninvariance of $\delta_{\mathbf{k}}$.

It is, however, necessary to specify the perturbations at a common time to carry out most calculations; e.g., an $N$-body simulation of structure formation or the calculation of CBR anisotropy. To do so, one has to take account of the evolution of 
the perturbations after they enter the horizon. After entering the horizon tensor perturbations behave like gravitons, with $h_{\mathbf{k}}$ decreasing as $R^{-1}$ and the energy density associated with a given mode, $\rho_{k} \sim m_{\mathrm{Pl}}^{2} k^{5}\left|h_{\mathbf{k}}\right|^{2} / R^{2}$, decreasing as $R^{-4}$. The evolution of scalar perturbations is slightly more complicated; modes that enter the horizon while the Universe is still radiation dominated remain essentially constant until the Universe becomes matter dominated (growing only logarithmically); modes that enter the horizon after the Universe becomes matter dominated grow as the scale factor. (The gauge noninvariance of $\delta_{\mathbf{k}}$ is not an important issue for subhorizon size modes; here a Newtonian analysis suffices, and there is only one growing mode, corresponding to a density perturbation.)

The method for characterizing the scalar perturbations is by now standard: The spectrum of perturbations is specified at the present epoch (assuming linear growth for all scales); the spectrum at earlier epochs can be obtained by multiplying $\delta_{\mathbf{k}}$ by $R(t) / R_{\text {today }}$. The inflationary metric perturbations are gaussian; thus $\delta_{\mathbf{k}}$ is a gaussian, random variable. Its statistical expectation value is

$$
\left\langle\delta_{\mathbf{k}} \delta_{\mathbf{q}}\right\rangle=P_{S}(k)(2 \pi)^{3} \delta^{(3)}(\mathbf{k}-\mathbf{q})
$$

where the power spectrum today is written as

$$
P_{S}(k) \equiv A k^{n} T(k)^{2}
$$

$n=1-2 \alpha_{S}$ (=1 for scale-invariant perturbations), and $T(k)$ is the "transfer function" which encodes the information about the post-horizon crossing evolution of each mode and depends upon the matter content of the Universe, e.g., baryons plus cold dark matter, baryons plus hot dark matter, baryons plus hot and cold dark matter, and so on. The transfer function is defined so that $T(k) \rightarrow 1$ for $k \rightarrow 0$ (long-wavelength perturbations); an analytic approximation to the cold dark matter transfer function is given by 74

$$
T(k)=\frac{\ln (1+2.34 q) / 2.34 q}{\left[1+(3.89 q)+(16.1 q)^{2}+(5.46 q)^{3}+(6.71 q)^{4}\right]^{1 / 4}} ;
$$

where $q=k /\left(\Omega_{0} h^{2} \mathrm{Mpc}^{-1}\right)$. Inflationary power spectra for different dark matter possibilities are shown in Fig. 9.

The overall normalization factor

$$
A=\frac{1024 \pi^{3}}{75 H_{0}^{3+n}} \frac{V_{50}}{m_{\mathrm{Pl}}^{4} x_{50}^{2}} \frac{\left[1+\frac{7}{6} n_{T}-\frac{1}{3}(n-1)\right]\left\{\Gamma\left[\frac{3}{2}-\frac{1}{2}(n-1)\right]\right\}^{2}}{2^{n-1}\left[\Gamma\left(\frac{3}{2}\right)\right]^{2}} k_{50}^{1-n}
$$

where the $\mathcal{O}\left(\alpha_{i}\right)$ correction to $A$ has been included [75]. The quantity $n_{T}=-2 \alpha_{T}=$ $-x_{50}^{2} / 8 \pi, n-1=-2 \alpha_{S}=n_{T}+x_{50}^{\prime} / 4 \pi, k_{50}$ is the comoving wavenumber of the scale that crossed outside the horizon 50 e-folds before the end of inflation. All the formulas below simplify if this scale corresponds to the present horizon scale, specifically, $k_{50}=$ 
Figure 9: Power spectra for cold dark matter (CDM), hot dark matter (HDM), mixed dark matter (MDM $=30 \%$ hot $+70 \%$ cold $)$, and cold dark matter with a cosmological constant $(\Lambda \mathrm{CDM}=20 \% \mathrm{CDM}+80 \% \Lambda)$. All spectra are normalized to the COBE DMR quadrupole temperature anisotropy; $h=0.5$ for all models except $\Lambda$ CDM $(h=0.8)$. 
$H_{0} / 2$. [Eq. (71) can be simplified by expanding $\Gamma\left(\frac{3}{2}+x\right)=\Gamma(3 / 2)[1+x(2-2 \ln 2-\gamma)]$, valid for $|x| \ll 1 ; \gamma \simeq 0.577$ is Euler's constant.]

From this expression it is simple to compute the Sachs-Wolfe contribution of scalar perturbations to the CBR temperature anisotropy; on angular scales much greater than about $1^{\circ}$ (corresponding to multipoles $l \ll 100$ ) it is the dominant contribution. If we expand the CBR temperature on the sky in spherical harmonics,

$$
\frac{\delta T(\theta, \phi)}{T_{0}}=\sum_{l \geq 2, m=-l}^{l=\infty, m=l} a_{l m} Y_{l m}(\theta, \phi) ;
$$

where $T_{0}=2.73 \mathrm{~K}$ is the CBR temperature today, then the ensemble expectation for the multipole coefficients is given by

$$
\begin{aligned}
\left\langle\left|a_{l m}\right|^{2}\right\rangle & =\frac{H_{0}^{4}}{2 \pi} \int_{0}^{\infty} k^{-2} P_{S}(k)\left|j_{l}\left(k r_{0}\right)\right|^{2} d k \\
& \simeq \frac{A H_{0}^{3+n} r_{0}^{1-n}}{16} \frac{\Gamma\left(l+\frac{1}{2} n-\frac{1}{2}\right) \Gamma(3-n)}{\Gamma\left(l-\frac{1}{2} n+\frac{5}{2}\right)\left[\Gamma\left(2-\frac{1}{2} n\right)\right]^{2}}
\end{aligned}
$$

where $r_{0} \approx 2 H_{0}^{-1}$ is the comoving distance to the last scattering surface, and this expression is for the Sachs-Wolfe contribution from scalar perturbations only. For $n$ not too different from one, the ensemble expectation for the quadrupole CBR temperature anisotropy is

$$
\left(\frac{\Delta T}{T_{0}}\right)_{Q-S}^{2} \equiv \frac{5\left|a_{2 m}\right|^{2}}{4 \pi} \approx \frac{32 \pi}{45} \frac{V_{50}}{m_{\mathrm{Pl}}^{4} x_{50}^{2}}\left(k_{50} r_{0}\right)^{1-n} .
$$

(By choosing $k_{50}=r_{0}^{-1}=\frac{1}{2} H_{0}$, the last factor becomes unity.)

The ensemble expectation values for the multipole amplitudes are often referred to as the angular power spectrum. Further, the rms temperature fluctuation on a given angular scale is related to the multipole amplitudes

$$
\left(\frac{\Delta T}{T}\right)_{\theta}^{2} \sim l^{2}\left\langle\left|a_{l m}\right|^{2}\right\rangle \quad \text { for } l \simeq 200^{\circ} / \theta
$$

The procedure for specifying the tensor modes is similar, cf. Refs. [76, 77]. For the modes that enter the horizon after the Universe becomes matter dominated, $k \lesssim$ $0.1 h^{2} \mathrm{Mpc}$, which are the only modes that contribute significantly to CBR anisotropy on angular scales greater than a degree,

$$
h_{\mathbf{k}}^{i}(\tau)=a^{i}(\mathbf{k})\left(\frac{3 j_{1}(k \tau)}{k \tau}\right)
$$

where $\tau=r_{0}\left(t / t_{0}\right)^{1 / 3}$ is conformal time. [For the modes that enter the horizon during the radiation-dominated era, $k \gtrsim 0.1 h^{2} \mathrm{Mpc}^{-1}$, the factor $3 j_{1}(k \tau) / k \tau$ is replaced by $j_{0}(k \tau)$ for the remainder of the radiation era. In either case, the factor involving the 
spherical Bessel function quantifies the fact that tensor perturbations remain constant while outside the horizon, and after horizon crossing decrease as $R^{-1}$.]

The tensor perturbations too are characterized by a gaussian, random variable, here written as $a^{i}(\mathbf{k})$; the statistical expectation

$$
\left\langle h_{\mathbf{k}}^{i} h_{\mathbf{q}}^{j}\right\rangle=P_{T}(k)(2 \pi)^{6} \delta^{(3)}(\mathbf{k}-\mathbf{q}) \delta_{i j}
$$

where the power spectrum

$$
\begin{aligned}
P_{T}(k) & =A_{T} k^{n_{T}-3}\left[\frac{3 j_{1}(k \tau)}{k \tau}\right]^{2} ; \\
A_{T} & =\frac{8}{3 \pi} \frac{V_{50}}{m_{\mathrm{Pl}}{ }^{4}} \frac{\left(1+\frac{5}{6} n_{T}\right)\left[\Gamma\left(\frac{3}{2}-\frac{1}{2} n_{T}\right)\right]^{2}}{2^{n_{T}}\left[\Gamma\left(\frac{3}{2}\right)\right]^{2}} k_{50}^{-n_{T}} ;
\end{aligned}
$$

where the $\mathcal{O}\left(\alpha_{i}\right)$ correction to $A_{T}$ has been included. Note that $n_{T}=-2 \alpha_{T}$ is zero for scale-invariant perturbations.

Finally, the contribution of tensor perturbations to the multipole amplitudes, which arise solely due to the Sachs-Wolfe effect [28, 76, 77], is given by

$$
\left\langle\left|a_{l m}\right|^{2}\right\rangle \simeq 36 \pi^{2} \frac{\Gamma(l+3)}{\Gamma(l-1)} \int_{0}^{\infty} k^{n_{T}+1} A_{T}\left|F_{l}(k)\right|^{2} d k
$$

where

$$
F_{l}(k)=-\int_{r_{D}}^{r_{0}} d r \frac{j_{2}(k r)}{k r}\left[\frac{j_{l}\left(k r_{0}-k r\right)}{\left(k r_{0}-k r\right)^{2}}\right] ;
$$

and $r_{D}=r_{0} /\left(1+z_{D}\right)^{1 / 2} \approx r_{0} / 35$ is the comoving distance to the horizon at decoupling (= conformal time at decoupling). Equation (81) is approximate in that very short wavelength modes, $k r_{0} \gg 100$, that crossed inside the horizon before matter-radiation equality have not been properly taken into account; to take them into account, the integrand must be multiplied by a transfer function,

$$
T(k) \simeq 1.0+1.44\left(k / k_{\mathrm{EQ}}\right)+2.54\left(k / k_{\mathrm{EQ}}\right)^{2} ;
$$

where $k_{\mathrm{EQ}} \equiv H_{0} /(2 \sqrt{2}-2) R_{\mathrm{EQ}}^{1 / 2}$ is the scale that entered the horizon at matter radiation equality [78]. In addition, for $l \gtrsim 1000$, the finite thickness of the lastscattering surface must be taken into account.

The tensor contribution to the quadrupole CBR temperature anisotropy for $n_{T}$ not too different from zero is

$$
\left(\frac{\Delta T}{T_{0}}\right)_{Q-T}^{2} \equiv \frac{5\left|a_{2 m}\right|^{2}}{4 \pi} \simeq 0.61 \frac{V_{50}}{m_{\mathrm{Pl}}{ }^{4}}\left(k_{50} r_{0}\right)^{-n_{T}} ;
$$

where the integrals in the previous expressions have been evaluated numerically.

Both the scalar and tensor contributions to a given multipole are dominated by wavenumbers $k r_{0} \sim l$. For scale-invariant perturbations and small $l$, both the scalar 
Figure 10: Scalar and tensor contributions to the CBR multipole moments: $l(l+$ $1)\left\langle\left|a_{l m}\right|^{2}\right\rangle / 6\left\langle\left|a_{2 m}\right|^{2}\right\rangle$ for the scalar and $l\left(l+\frac{1}{2}\right)\left\langle\left|a_{l m}\right|^{2}\right\rangle / 5\left\langle\left|a_{2 m}\right|^{2}\right\rangle$ for the tensor. The tensor contribution begins to fall off for $l \sim 30$; here $n-1=n_{T}=0, z_{\mathrm{DEC}}=1000$, and $h=0.5$ (from [79]).

and tensor contributions to $\left(l+\frac{1}{2}\right)^{2}\left\langle\left|a_{l m}\right|^{2}\right\rangle$ are approximately constant. The contribution of scalar perturbations to $\left(l+\frac{1}{2}\right)^{2}\left\langle\left|a_{l m}\right|^{2}\right\rangle$ begins to decrease for $l \sim 150$ because the scalar contribution to these multipoles is dominated by modes that entered the horizon before matter domination (and hence are suppressed by the transfer function). The contribution of tensor modes to $\left(l+\frac{1}{2}\right)^{2}\left\langle\left|a_{l m}\right|^{2}\right\rangle$ begins to decrease for $l \sim 30$ because the tensor contribution to these multipoles is dominated by modes that entered the horizon before decoupling (and hence decayed as $R^{-1}$ until decoupling). Figure 10 shows the contribution of scalar and tensor perturbations to the CBR anisotropy multipole amplitudes (and includes both the tensor and scalar transfer functions); the expected variance in the CBR multipoles is given by the sum of the scalar and tensor contributions. 


\subsection{Worked examples}

In this Section I apply the formalism developed in the two previous sections to four specific models. So that I can, where appropriate, solve numerically for model parameters, I will: (1) Assume that the astrophysically interesting scales crossed outside the horizon 50 e-folds before the end of inflation; and (2) Use the COBE DMR quadrupole measurement, $\left\langle(\Delta T)_{Q}^{2}\right\rangle^{1 / 2} \approx 16 \pm 2 \mu \mathrm{K}[1]$, to normalize the scalar perturbations; using Eq. (62) this implies

$$
V_{50} \approx 1.6 \times 10^{-11} m_{\mathrm{Pl}}^{4} x_{50}^{2}
$$

Of course it is entirely possible that a significant portion of the quadrupole anisotropy is due to tensor-mode perturbations, in which case this normalization must be reduced by a factor of $(1+T / S)^{-1}$. And, it is straightforward to change " 50 " to the number appropriate to a specific model, or to normalize the perturbations another way.

Before going on let us use the COBE DMR quadrupole anisotropy to bound the tensor contribution to the quadrupole anisotropy and thereby the energy density that drives inflation:

$$
V_{50} \lesssim 6 \times 10^{-11} m_{\mathrm{Pl}}^{4}
$$

Thus, the tensor contribution to the CBR quadrupole implies that the vacuum energy that drives inflation must be much less than the Planck energy density, strongly suggesting that inflation is not a quantum-gravitational phenomenon.

\subsubsection{Exponential potentials}

There are a class of models that can be described in terms of an exponential potential,

$$
V(\phi)=V_{0} \exp \left(-\beta \phi / m_{\mathrm{Pl}}\right)
$$

This type of potential was first invoked in the context of power-law inflation [80], and has recently received renewed interest in the context of extended inflation [44]. In the simplest model of extended, or first-order, inflation, that based upon the BransDicke-Jordan theory of gravity [44], $\beta$ is related to the Brans-Dicke parameter: $\beta^{2}=$ $64 \pi /(2 \omega+3)$.

For such a potential the slow-roll conditions are satisfied provided that $\beta^{2} \lesssim 24 \pi$; thus inflation does not end until the potential changes shape, or in the case of extended inflation, until the phase transition takes place. In either case we can relate $\phi_{50}$ to $\phi_{\text {end }}$,

$$
N\left(\phi_{50}\right)=50=\frac{8 \pi}{m_{\mathrm{Pl}}^{2}} \int_{\phi_{50}}^{\phi_{\mathrm{end}}} \frac{V d \phi}{-V^{\prime}} ; \quad \Rightarrow \quad \phi_{50}=\phi_{\mathrm{end}}-50 \beta / 8 \pi .
$$

Since $\phi_{\text {end }}$ is in effect arbitrary, the overall normalization of the potential is irrelevant. The two other parameters, $x_{50}$ and $x_{50}^{\prime}$, are easy to compute:

$$
x_{50}=-\beta ; \quad x_{50}^{\prime}=0 .
$$


Using the COBE DMR normalization, we can relate $V_{50}$ and $\beta$ :

$$
V_{50}=1.6 \times 10^{-11} m_{\mathrm{Pl}}^{4} \beta^{2} .
$$

Further, we can compute $q, \alpha_{S}, \alpha_{T}$, and $T / S$ :

$$
q=16 \pi / \beta^{2} ; \quad T / S=0.28 \beta^{2} ; \quad \alpha_{T}=\alpha_{S}=1 /(q-1) \simeq \beta^{2} / 16 \pi .
$$

Note, for the exponential potential, $q, \alpha_{T}=\alpha_{S}$ are independent of epoch. In the case of extended inflation, $\alpha_{S}=\alpha_{T}=4 /(2 \omega+3)$; since $\omega$ must be less than about 20 [47], this implies significant tilt: $\alpha_{S}=\alpha_{T} \gtrsim 0.1$.

\subsubsection{Chaotic inflation}

These models are based upon a very simple potential:

$$
V(\phi)=a \phi^{b}
$$

$b=4$ corresponds to Linde's original model of chaotic inflation and $a$ is dimensionless "54], and $b=2$ is a model based upon a massive scalar field and $m^{2}=2 a$ [81]. In these models $\phi$ is initially displaced from $\phi=0$, and inflation occurs as $\phi$ slowly rolls to the origin. The value of $\phi_{\mathrm{end}}$ is easily found: $\phi_{\mathrm{end}}^{2}=b(b-1) m_{\mathrm{Pl}}^{2} / 24 \pi$, and

$$
\begin{aligned}
N\left(\phi_{50}\right)=50 & =\frac{8 \pi}{m_{\mathrm{Pl}}^{2}} \int_{\phi_{\mathrm{end}}}^{\phi_{50}} \frac{V d \phi}{V^{\prime}} ; \\
& \Rightarrow \phi_{50}^{2} / m_{\mathrm{Pl}}^{2}=50 b / 4 \pi+b^{2} / 48 \pi \simeq 50 b / 4 \pi ;
\end{aligned}
$$

the value of $\phi_{50}$ is a few times the Planck mass.

For purposes of illustration consider $b=4 ; \phi_{\mathrm{end}}=m_{\mathrm{Pl}} / \sqrt{2 \pi} \simeq 0.4 m_{\mathrm{Pl}}, \phi_{50} \simeq 4 m_{\mathrm{Pl}}$, $\phi_{46} \simeq 3.84 m_{\mathrm{Pl}}$, and $\phi_{54} \simeq 4.16 \mathrm{~m}_{\mathrm{Pl}}$. In order to have sufficient inflation the initial value of $\phi$ must exceed about $4.2 m_{\mathrm{Pl}}$; inflation ends when $\phi \approx 0.4 m_{\mathrm{Pl}}$; and the scales of astrophysical interest cross outside the horizon over an interval $\Delta \phi \simeq 0.3 m_{\mathrm{Pl}}$. found,

The values of the potential, its steepness, and the change in steepness are easily

$$
\begin{gathered}
V_{50}=a m_{\mathrm{Pl}}^{b}\left(\frac{50 b}{4 \pi}\right)^{b / 2} ; \quad x_{50}=\sqrt{\frac{4 \pi b}{50}} ; \quad m_{\mathrm{Pl}} x_{50}^{\prime}=\frac{-4 \pi}{50} \\
q_{50}=200 / b ; \quad T / S=0.07 b ; \quad \alpha_{T} \simeq b / 200 ; \quad \alpha_{S}=\alpha_{T}+0.01 .
\end{gathered}
$$

Unless $b$ is very large, scalar perturbations dominate tensor perturbations [82], $\alpha_{T}$, $\alpha_{S}$ are very small, and $q$ is very large. Further, when $\alpha_{T}, \alpha_{S}$ become significant, they are equal. Using the COBE DMR normalization we find:

$$
a=1.6 \times 10^{-11} b^{1-b / 2}(4 \pi / 50)^{b / 2+1} m_{\mathrm{Pl}}^{4-b} .
$$

For the two special cases of interest: $b=4, a=6.4 \times 10^{-14}$; and $b=2, m^{2} \equiv 2 a=$ $2.0 \times 10^{-12} \mathrm{~m}_{\mathrm{Pl}}^{2}$. 


\subsubsection{New inflation}

These models entail a very flat potential where the scalar field rolls from $\phi \approx 0$ to the minimum of the potential at $\phi=\sigma$. The original models of slow-rollover inflation [83] were based upon potentials of the Coleman-Weinberg form

$$
V(\phi)=B \sigma^{4} / 2+B \phi^{4}\left[\ln \left(\phi^{2} / \sigma^{2}\right)-\frac{1}{2}\right] ;
$$

where $B$ is a very small dimensionless coupling constant. Other very flat potentials also work (e.g., $V=V_{0}-\alpha \phi^{4}+\beta \phi^{6}[72]$ ). As before we first solve for $\phi_{50}$ :

$$
N\left(\phi_{50}\right)=50=\frac{8 \pi}{m_{\mathrm{Pl}}^{2}} \int_{\phi_{\mathrm{end}}}^{\phi_{50}} \frac{V d \phi}{V^{\prime}} ; \quad \Rightarrow \quad \phi_{50}^{2}=\frac{\pi \sigma^{4}}{100\left|\ln \left(\phi_{50}^{2} / \sigma^{2}\right)\right| m_{\mathrm{Pl}}^{2}}
$$

where the precise value of $\phi_{\text {end }}$ is not relevant, only the fact that it is much larger than $\phi_{50}$. Provided that $\sigma \lesssim m_{\mathrm{Pl}}$, both $\phi_{50}$ and $\phi_{\text {end }}$ are much less than $\sigma$; we then find

$$
\begin{gathered}
V_{50} \simeq B \sigma^{4} / 2 ; \quad x_{50} \simeq-\frac{(\pi / 25)^{3 / 2}}{\sqrt{\mid \ln \left(\phi_{50}^{2} / \sigma^{2} \mid\right)}}\left(\frac{\sigma}{m_{\mathrm{Pl}}}\right)^{2} \ll 1 ; \\
m_{\mathrm{Pl}} x_{50}^{\prime} \simeq-24 \pi / 100 ; \quad q_{50} \simeq \frac{2.5 \times 10^{5}\left|\ln \left(\phi_{50}^{2} / \sigma^{2}\right)\right|}{\pi^{2}}\left(\frac{m_{\mathrm{Pl}}}{\sigma}\right)^{4} \gg 1 ; \\
\alpha_{S} \simeq \frac{1}{q_{50}} \ll 1 ; \quad \alpha_{T}=\alpha_{S}+0.03 ; \quad \frac{T}{S} \simeq \frac{6 \times 10^{-4}}{\left|\ln \left(\phi_{50}^{2} / \sigma^{2}\right)\right|}\left(\frac{\sigma}{m_{\mathrm{Pl}}}\right)^{4} .
\end{gathered}
$$

Provided that $\sigma \lesssim m_{\mathrm{Pl}}, x_{50}$ is very small; this means that $q$ is very large, gravitywaves and density perturbations are very nearly scale invariant, and $T / S$ is small. Finally, using the COBE DMR normalization, we can determine the dimensionless coupling constant $B$ :

$$
B \simeq 6 \times 10^{-14} /\left|\ln \left(\phi_{50}^{2} / \sigma^{2}\right)\right| \approx 3 \times 10^{-15} .
$$

\subsubsection{Natural inflation}

This model is based upon a potential of the form 53

$$
V(\phi)=\Lambda^{4}[1+\cos (\phi / f)] .
$$

The flatness of the potential (and requisite small couplings) arise because the $\phi$ particle is a pseudo-Nambu-Goldstone boson ( $f$ is the scale of spontaneous symmetry breaking and $\Lambda$ is the scale of explicit symmetry breaking; in the limit that $\Lambda \rightarrow 0$ the $\phi$ particle is a massless Nambu-Goldstone boson). It is a simple matter to show that $\phi_{\text {end }}$ is of the order of $\pi f$.

This potential is difficult to analyze in general; however, there are two limiting regimes: (i) $f \gg m_{\mathrm{Pl}}$; and (ii) $f \lesssim m_{\mathrm{Pl}}$ [72]. In the first regime, the 50 or so relevant 
e-folds take place close to the minimum of the potential, $\sigma=\pi f$, and inflation can be analyzed by expanding the potential about $\phi=\sigma$,

$$
\begin{gathered}
V(\psi) \simeq m^{2} \psi^{2} / 2 ; \\
m^{2}=\Lambda^{4} / f^{2} ; \quad \psi=\phi-\sigma .
\end{gathered}
$$

In this regime natural inflation is equivalent to chaotic inflation with $m^{2}=\Lambda^{4} / f^{2} \simeq$ $2 \times 10^{-12} m_{\mathrm{Pl}}^{2}$.

In the second regime, $f \lesssim m_{\mathrm{Pl}}$, inflation takes place when $\phi \lesssim \pi f$, so that we can make the following approximations: $V \simeq 2 \Lambda^{4}$ and $V^{\prime}=-\Lambda^{4} \phi / f^{2}$. Taking $\phi_{\text {end }} \sim \pi f$, we can solve for $N(\phi)$ :

$$
N(\phi)=\frac{8 \pi}{m_{\mathrm{Pl}}^{2}} \int_{\phi}^{\pi f} \frac{V d \phi}{-V^{\prime}} \simeq \frac{16 \pi m_{\mathrm{Pl}}^{2}}{f^{2}} \ln (\pi f / \phi) ;
$$

from which it is clear that achieving 50 e-folds of inflation places a lower bound to $f$, very roughly $f \gtrsim m_{\mathrm{Pl}} / 3[72,53]$.

Now we can solve for $\phi_{50}, V_{50}, x_{50}$, and $x_{50}^{\prime}$ :

$$
\begin{aligned}
\phi_{50} / \pi f & \simeq \exp \left(-50 m_{\mathrm{Pl}}^{2} / 16 \pi f^{2}\right) \lesssim \mathcal{O}(0.1) ; \quad V_{50} \simeq 2 \Lambda^{4} ; \\
x_{50} & \simeq \frac{1}{2} \frac{m_{\mathrm{Pl}}}{f} \frac{\phi_{50}}{f} \lesssim \mathcal{O}(0.1) ; \quad x_{50}^{\prime} \simeq-\frac{1}{2}\left(\frac{m_{\mathrm{Pl}}}{f}\right)^{2} .
\end{aligned}
$$

Using the COBE DMR normalization, we can relate $\Lambda$ to $f / m_{\mathrm{Pl}}$ :

$$
\Lambda / m_{\mathrm{Pl}}=6.7 \times 10^{-4} \sqrt{\frac{m_{\mathrm{Pl}}}{f}} \exp \left(-25 m_{\mathrm{Pl}}^{2} / 16 \pi f^{2}\right) .
$$

Further, we can solve for $T / S, \alpha_{T}$, and $\alpha_{S}$ :

$$
\begin{gathered}
\frac{T}{S} \simeq 0.07\left(\frac{m_{\mathrm{Pl}}}{f}\right)^{2}\left(\frac{\phi_{50}}{f}\right)^{2} \lesssim \mathcal{O}(0.1) \\
\alpha_{T}=\frac{1}{16 \pi} \frac{q_{50}}{q_{50}-1}\left(\frac{1}{4} \frac{m_{\mathrm{Pl}}^{2}}{f^{2}} \frac{\phi_{50}^{2}}{f^{2}}\right) \approx \frac{1}{64 \pi}\left(\frac{m_{\mathrm{Pl}}}{f}\right)^{2}\left(\frac{\phi_{50}}{f}\right)^{2} \ll 0.1 ; \\
\alpha_{S}=\frac{1}{16 \pi} \frac{q_{50}}{q_{50}-1}\left(\frac{1}{4} \frac{m_{\mathrm{Pl}}^{2}}{f^{2}} \frac{\phi_{50}^{2}}{f^{2}}+\frac{m_{\mathrm{Pl}}^{2}}{f^{2}}\right) \approx \frac{1}{16 \pi}\left(\frac{m_{\mathrm{Pl}}}{f}\right)^{2} ; \\
q_{50}=64 \pi\left(\frac{f}{m_{\mathrm{Pl}}}\right)^{2}\left(\frac{f}{\phi_{50}}\right)^{2} \gg 1 .
\end{gathered}
$$

Regime (ii) provides the exception to the rule that $\alpha_{S} \approx \alpha_{T}$ and large $\alpha_{S}$ implies large $T / S$. For example, taking $f=m_{\mathrm{Pl}} / 2$, we find:

$$
\phi_{50} / f \sim 0.06 ; \quad x_{50} \sim 0.06 ; \quad x_{50}^{\prime}=-2 ; \quad q_{50} \sim 10^{4} ;
$$




$$
\alpha_{T} \sim 10^{-4} ; \quad \alpha_{S} \sim 0.08 ; \quad T / S \sim 10^{-3}
$$

The gravitational-wave perturbations are very nearly scale invariant, while the density perturbations deviate significantly from scale invariance. I note that regime (ii), i.e., $f \lesssim m_{\mathrm{Pl}}$, occupies only a tiny fraction of parameter space because $f$ must be greater than about $m_{\mathrm{Pl}} / 3$ to achieve sufficient inflation; further, regime (ii) is "fine tuned" and "unnatural" in the sense that the required value of $\Lambda$ is exponentially sensitive to the value of $f / m_{\mathrm{Pl}}$.

Finally, I note that the results for regime (ii) apply to any inflationary model whose Taylor expansion in the inflationary region is similar; e.g., $V(\phi)=-m^{2} \phi^{2}+\lambda \phi^{4}$, which was originally analyzed in Ref. [72].

\subsubsection{Lessons}

To summarize the general features of our results. In all examples the deviations from scale invariance enhance perturbations on large scales. The only potentials that have significant deviations from scale invariance are either very steep or have rapidly changing steepness. In the former case, both the scalar and tensor perturbations are tilted by a similar amount; in the latter case, only the scalar perturbations are tilted.

For "steep" potentials, the expansion rate is "slow," i.e., q50 close to unity, the gravity-wave contribution to the CBR quadrupole anisotropy becomes comparable to, or greater than, that of density perturbations, and both scalar and tensor perturbations are tilted significantly. For flat potentials, i.e., small $x_{50}$, the expansion rate is "fast," i.e., $q_{50} \gg 1$, the gravity-wave contribution to the CBR quadrupole is much smaller than that of density perturbations, and unless the steepness of the potential changes significantly, large $x_{50}^{\prime}$, both spectra very nearly scale invariant; if the steepness of the potential changes rapidly, the spectrum of scalar perturbations can be tilted significantly. The models that permit significant deviations from scale invariance involve exponential or low-order polynomial potentials; the former by virtue of their steepness, the latter by virtue of the rapid variation of their steepness. Exponential potentials are of interest because they arise in extended inflation models; potentials with rapidly steepness include $V(\phi)=-m^{2} \phi^{2}+\lambda \phi^{4}$ or $\Lambda^{4}[1+\cos (\phi / f)]$.

Finally, to illustrate how observational data could used to determine the properties of the inflationary potential and test the consistency of the inflationary hypothesis, suppose observations determined the following:

$$
(\Delta T)_{Q} \simeq 16 \mu \mathrm{K} ; \quad T / S=0.24 ; \quad n=0.9
$$

that is, the COBE DMR quadrupole anisotropy, a four to one ratio of scalar to tensor contribution to the CBR quadrupole, and spectral index of 0.9 for the scalar perturbations. From $T / S$, we determine the steepness of the potential: $x_{50} \simeq 0.94$.

From the steepness and the quadrupole anisotropy the value of the potential: $V_{50}^{1 / 4} \simeq$ $2.4 \times 10^{16} \mathrm{GeV}$. From the spectral index the change in steepness: $x_{50}^{\prime} \simeq-0.81 / m_{\mathrm{Pl}}$. These data can also be expressed in terms of the value of the potential and its first 
two derivatives:

$$
V_{50}=1.4 \times 10^{-11} m_{\mathrm{Pl}}^{4} ; \quad V_{50}^{\prime}=1.5 \times 10^{-11} m_{\mathrm{Pl}}^{3} ; \quad V_{50}^{\prime \prime}=1.0 \times 10^{-12} m_{\mathrm{Pl}}^{2} .
$$

Further, they the lead to the prediction: $n_{T}=-0.035$, which, when "measured," can be used as a consistency check for inflation.

\section{Structure Formation After COBE}

Filling in the details of structure formation is one of the pressing challenges of the standard cosmology. In order to do so one must have the "initial data" for the structure formation problem: the spectrum of density perturbations and the quantity and composition of matter in the Universe. With initial data in hand one can hope to carry out detailed numerical simulations which can be compared to the observations.7 While neither the observational data nor the simulations are perfect, the situation in both regards is improving rapidly. In particular, the discovery of CBR anisotropy by the COBE DMR has provided the first direct evidence for the existence of density perturbations and thereby opened the door for their study.

Over the past decade or so many cosmologists have come to believe that required initial data trace to events that took place during the earliest history of the Universe $\left(t \ll 10^{-2} \mathrm{sec}\right)$. Thus, the study of structure formation has the potential to test theories of the early Universe and the underlying particle physics. Inflation leads to two limiting scenarios: hot dark matter and cold dark matter, both with scaleinvariant density perturbations.

In the hot dark matter scenario the streaming of neutrinos from regions of higher density to lower density erases perturbations on small scales $\left(\lesssim 13 h^{-2} \mathrm{Mpc}\right)$; therefore structure forms from the the top down: superclusters must form first and fragment into smaller objects. Therein lies the fundamental problem: Since we know that superclusters are just forming today, galaxies form too late to be consistent with the abundance of galaxies observed at red shifts of unity or so 84].

Cold dark matter looks much more promising; cold dark matter refers to dark matter particles that move very slowly, either by virtue of their large mass (e.g., 10 $\mathrm{GeV}$ to $2 \mathrm{TeV}$ neutralino) or the fact that they were born cold $\left(10^{-5} \mathrm{eV}\right.$ axion). This means that perturbations on small scales are not erased and that structure forms from the bottom up. Cold dark matter has been subject to intense scrutiny over the past decade and has thus far survived, albeit with a number of scratches and bruises 85. CDM models will be the focus of this Section.

That is not to say that cold dark matter models are the only promising possibilities. There are scenarios where the density perturbations arise due to topological

\footnotetext{
${ }^{7}$ Since the fluctuations predicted by inflation and other theories are only specified in a statistical sense this comparison can only be done statistically; in the case of inflation, the fluctuations are gaussian and so all predictions can be specified in terms of the power spectrum, $\left\langle\left|\delta_{k}\right|^{2}\right\rangle$.
} 
(and nontopological) defects such as strings [30, global monopoles, and textures [31] with hot or cold dark matter. Scenarios have been discussed where the density perturbations arise in a rather recent phase transition (since decoupling!), due to new physics in the neutrino sector [32].

Finally, perhaps the most interesting alternative is Peebles' PIB model or whatyou-see-is-what-you-get model [29]. In PIB $\Omega_{0}=\Omega_{B} \sim 0.2, h \sim 0.8$, and the density perturbations are isocurvature perturbations (variations in the local baryon-to-photon ratio and not the energy density). PIB is not motivated by what early Universe theorists would like, rather by "what we see" (though it violates the primordial nucleosynthesis bound by large factor since $\Omega_{B} h^{2} \simeq 0.13 \gg 0.02$ ). Remarkably, the scenario is still viable, though measurements of CBR anisotropy on scales of $1^{\circ}$ to $90^{\circ}$ are really putting it to the test: normalizing to the COBE $10^{\circ}$ measurement, its predictions for the quadrupole are a factor of two small, while its predictions of scales of about five degrees exceed current upper limits [86].

\subsection{The Universe observed}

By now we know a lot - and a little - about the structure that exists in the Universe today. A resurgence of interest in structure formation, brought about in part by the very intriguing early Universe suggestions for initial data, has resulted in an explosion of observations that bear on the issue over the past decade. They include red-shift surveys (large-angle, pencil-beam and sparsely sampled surveys), the spectrum and spatial variation of the CBR temperature, peculiar-velocity measurements, QSO absorption line systems, studies of clusters and superclusters, determinations of the distribution and quantity of dark matter, studies of galactic evolution, catalogues of millions of galaxies on the sky, and on and on.

To place things in perspective, we know much about the distribution of light (bright galaxies) - as opposed to mass (which is what theorists like to discuss); the largest red-shift survey, the $\mathrm{CfA}_{2}$ slices of the Universe, contains only about 20,000 galaxies with median red shift of about 0.02 [87]; and the total number of red shifts measured for all purposes is only about 50,000. We have no definitive evidence as to the epoch of galaxy formation, or how the neutral hydrogen left between galaxies became ionized (if it weren't, it we would not be able to see emission from distant QSOs shortward of Lyman alpha, $1215 \AA$ in the rest frame of the QSO). We probably only know the mean density of galaxies to within $20 \%$; we have no fair sample of clusters; and so on.

Let me briefly try to summarize some of the data that can be used to test models

of structure formation. Within the spirit of my broad brush description, I will group the observations into three classes: Small-scale, observations that probe the Universe on scales less than order $30 h^{-1} \mathrm{Mpc}$ or so; Intermediate-scale, observations that probe the Universe on scales of $30 h^{-1} \mathrm{Mpc}-300 h^{-1} \mathrm{Mpc}$ or so; and Large-scale, observations 
that probe the Universe on the very largest scales accessible.f

- Small-scale Structure $\left(\lambda \lesssim 30 h^{-1} \mathrm{Mpc}\right)$ : Our knowledge of these scales is the most extensive and well developed, though largely restricted to the distribution of bright galaxies like our own. These are also the scales on which astrophysical effects - star formation, blast waves, and so on - are potentially most important and poorly understood. The Universe on these scales is organized into galaxies and clusters, whose properties have been studied and quantified; for galaxies, number density and morphology-i.e., spiral, elliptical, etc.-rotation curves, and so on; and for clusters, number density, velocity dispersions, richness class, and so on. Both galaxies and clusters cluster, with measured two-point correlation functions, $\xi_{\mathrm{gg}}(r) \simeq\left(r / 5 h^{-1} \mathrm{Mpc}\right)^{-1.8}$ and $\xi_{\mathrm{cc}}(r) \simeq\left(r / 25 h^{-1} \mathrm{Mpc}\right)^{-1.8}$, though the cluster correlation function is less well known and depends upon cluster richness 88. At some level we know the distribution of dark matter: spiral galaxies have large halos with unknown spatial extent and the bulk of the mass in clusters is dark [17]. We also know the pairwise galaxy velocity dispersion (line-of-sight velocity dispersion), $\left.\left\langle\left(v_{1}-v_{2}\right)^{2}\right\rangle^{1 / 2}\right|_{10 \mathrm{Mpc}} \simeq 300-400 \mathrm{~km} \mathrm{~s}^{-1}$ [89]. (As discussed earlier, the peculiar motions of galaxies depend upon the amplitude of density perturbations and the amount of matter in the Universe, and thus are indicative of such.) On scales less than about $8 h^{-1} \mathrm{Mpc}$ the Universe is nonlinear: specifically, the rms fluctuation in the number density of bright galaxies measured in a sphere of radius of $8 h^{-1} \mathrm{Mpc}$ is unity.

- Intermediate-scale Structure $\left(30 h^{-1} \mathrm{Mpc}-300 h^{-1} \mathrm{Mpc}\right)$ : These are the scales on which our knowledge is the most fragmentary and often more qualitative than quantitative. P' Observations include the the voids and "Great Wall" seen in the $\mathrm{CfA}_{2}$ red shift survey; the reoccurring walls seen in the pencil-beam survey of Broadhurst et al. [90; the angular-correlation function of galaxies $w(\theta)$, which is related to $\xi_{g g}(r)$, measured by Efstathiou et al. [91] in the APM catalogue of 2 million galaxies on the sky (effective depth of $400 h^{-1} \mathrm{Mpc}$ ); the peculiar velocities of galaxies measured by the Seven Samurai and others [92, about $400 \mathrm{~km} \mathrm{~s}^{-1}$ on the scale of $50 h^{-1} \mathrm{Mpc}$; Great Attractors, and on and on. From red-shift surveys like the $\mathrm{CfA}_{2}$ slices of the Universe, the IRAS 1.2 Jy survey of infrared-selected galaxies [93, and the APM-Stromlo 1 in 20 red-shift survey [94], the fluctuations in the galaxy number density have been measured on scales out to a few hundred Mpc; see Fig. 11a. By the year 2000 the Sloan Digital Sky Survey [95] will produce a "Map of the Universe," from the red shifts of a million galaxies (mean red shift of about 0.15 and survey depth of $500 h^{-1} \mathrm{Mpc}$ ).

\footnotetext{
${ }^{8} \mathrm{I}$ warn the reader that my nomenclature is not universal; many refer to what I call intermediate scales as large scales.

${ }^{9}$ I often call these the $N Y$ Times scales, as new observations and their extravagant interpretation are reported there almost weekly!
} 
With the exception of the peculiar-velocity measurements all these observations probe the distribution of light not mass. CBR anisotropy measurements on angular scales of a few degrees down to a few arcminutes also have the potential to probe the distribution of matter on these scales, as the CBR anisotropy on a given angular scale is related to the fluctuations in the mass density on a ranges of length scales around the characteristic length scale that subtends that angular size on the last scattering surface: $\lambda \sim 100 h^{-1} \mathrm{Mpc}(\theta / \mathrm{deg})$. Very sensitive experiments are being done on these angular scales; with the important exception of the COBE DMR detection, there are now only upper limits, at the level of a few times $10^{-5}$; see Fig. 3. I believe that more detections are just around the corner!

- Large-scale Structure $\left(\gtrsim 300 h^{-1} \mathrm{Mpc}\right)$ : These scales are probed primarily by CBR anisotropy, though the Sloan Digital Sky Survey should provide some information about the distribution of galaxies on these scales. On angular scales much greater than about $1^{\circ}$ the anisotropy arises due to the fluctuations in the gravitational potential on the last-scattering surface (Sachs-Wolfe effect), while on small-angular scales the situation is more complicated as the velocity of the matter, temperature fluctuations intrinsic to the radiation, and the ionization history of the Universe become important. On large-angular scales it is very simple to relate the CBR anisotropy to the "virgin spectrum" of density fluctuations. It is these scales that were probed by the COBE DMR detection, proving the first direct information about the existence of the density inhomogeneity that seeded structure formation.

\subsubsection{Normalization: the great leap forward!}

Lacking a definite prediction for the overall normalization for inflationary density perturbations, those who study formation of structure have historically used data on small-scales to normalize the spectrum of density perturbations, typically on the scale of $8 h^{-1} \mathrm{Mpc}$. In so doing it is useful to define

$$
\sigma_{8} \equiv\left\langle(\delta M / M)^{2}\right\rangle_{8 h^{-1} \mathrm{Mpc}}^{1 / 2}
$$

which is the rms mass fluctuation in spheres of radius $8 h^{-1} \mathrm{Mpc}$. The simplest (and most naive) procedure is to assume that light faithfully traces mass, i.e., $\delta \rho / \rho=$ $\delta n_{\mathrm{GAL}} / n_{\mathrm{GAL}}$, and set $\sigma_{8}=1$ since the rms fluctuation in galaxy number in spheres of radius $8 h^{-1} \mathrm{Mpc}$ is unity; I will refer to this minimal cold dark matter (MCDM). I should remark that there is no a priori reason to expect light to trace mass, except on the very largest scales where only gravity is important.

Because this normalization leads to a galaxy pairwise velocity dispersion that is about a factor of two too large, the concept of "biasing" was introduced; namely, that light is a biased tracer of mass [96]. If light doesn't trace mass, the simplest ansatz is a linear factor between the two:

$$
\delta n_{\mathrm{GAL}} / n_{\mathrm{GAL}}=b(\delta \rho / \rho) .
$$


Figure 11: Summary of observational knowledge of the power spectrum $\left|\delta_{k}\right|^{2}$ based upon the IRAS 1.2 Jy red shift survey and CBR anisotropy measurements (from 93]). ACME-HEMT indicates the South Pole experiment that has detected anisotropy that may or may not be intrinsic to the CBR.

Of course there is every reason to expect that the real relationship is more complicated, $b=b(\lambda)$. In biased CDM models (BCDM), $\sigma_{8}=b^{-1}$. In principle, the bias factor $b(\lambda)$ can be measured on scales where there is information about both the distribution of galaxies and of mass, cf. Fig. 11.

Until the COBE DMR detection, a bias factor to 1.5 to 2 was in vogue to resolve the discrepancy in the galaxy pairwise velocity dispersion; $b \sim 1.5-2$ was known as the standard CDM model. Since the peculiar velocities of galaxies arise due to the lumpy distribution of matter; larger $b$ implies a smoother mass distribution and thus smaller peculiar velocities. (Likewise, reducing the matter content, or $\Omega_{0}$, can help.) Unfortunately, the predictions of BCDM on intermediate-scales could not account for the level of inhomogeneity seen-voids, galaxy-galaxy angular-correlation function, peculiar velocities, and so on - since the mass distribution was smoother. Thus, CDM was faulted for predicting too little power on "large scales" (in my nomenclature, intermediate scales).

[Another motivation for bias is the so-called $\Omega$ problem: Why do the dynamical measurements indicate $\Omega_{0}=0.2$, if $\Omega_{0}$ is really unity? The biasing explanation is 
that most of the mass in the Universe is in low surface-brightness galaxies that are too faint to see and that are less strongly clustered than the bright galaxies. Bright galaxies are more strongly clustered and account for only $20 \%$ of the mass density.]

The COBE DMR detection of CBR anisotropy changed the situation overnight by providing a new, more direct normalization of the density perturbations! Assuming the correctness of the result, we now have a measurement of the inhomogeneity in the mass distribution on large-scales - and at last a "physics normalization." Remarkably, the COBE normalization (with scale-invariant perturbations) corresponds to the simplest CDM model: $\sigma_{8}=1.2 \pm 0.2$ [97, i.e., no biasing. "r]

The COBE DMR normalization has changed the way we view inflation and structure formation: Intermediate (and large) scales seem to be OK; the problem is with small scales. Addressing this problem is the focus of the brief discussion of CDM models that follows.

\subsection{CDM models}

The initial data for structure formation include: (i) spectrum of primeval density perturbations - amplitude on a given scale (normalization) and spectral index $n$; (ii) composition of the Universe $-\Omega_{i}, i=$ baryons, cold dark matter, hot dark matter, vacuum energy and so on; and (iii) Hubble constant which sets the time/length scale for the Universe. With these in hand one can compute the spectrum of density perturbations at the equivalence epoch and let gravity run its course. Of course, astrophysics - cooling of baryons, star formation, etc. - is important too, but more difficult to model. Progress here too is being made with large $N$-body codes that include both gravity and hydrodynamics for the baryons [98. The list of wanted cosmological parameters for a numerical simulation is: $\sigma_{8}$ (in the simple biasing prescription $\left.\sigma_{8}=b^{-1}\right), n, \Omega_{B}, \Omega_{\text {other }}$, and $h$.

What predictions does inflation make for these parameters? The firmest is a flat Universe, in my notation $\Omega_{0}=1.0$, which implies nonbaryonic dark matter dominates. As mentioned earlier, hot dark matter $(30 \mathrm{eV}$ or so neutrinos) was ruled out early on; and so the cold dark matter scenario appeared to be the unique inflationary blueprint for structure formation [99]. Let me explain; to get the age of the Universe right we must have $h \sim 0.5$. This fact together with the primordial nucleosynthesis determination of $\Omega_{B} h^{2}$ implies $\Omega_{B} \simeq 0.04-0.10$. In most inflationary models the density perturbations are very nearly scale invariant, implying $n=1$. Finally, the variance in galaxy counts on $8 h^{-1} \mathrm{Mpc}$ suggests $\sigma_{8}=1$. This is the minimal cold dark matter model (MCDM); it is certainly the simplest CDM scenario, though it is no longer the unique CDM model.

Partly due to problems with MCDM, partly due to the improvement in the observations that test models of structure formation, and partly due to the passage of

\footnotetext{
${ }^{10}$ For HDM the COBE DMR normalization implies $\sigma_{8}=0.7$. This drives another nail in the coffin, as it implies that only about $1 \%$ of the material in the Universe is in nonlinear structures.
} 
Figure 12: MCDM vs. observation.

time we now realize that there are other possibilities, some just as well motivated, some less well motivated. I will characterize the different models by their values for the key cosmological parameters for structure formation: $\sigma_{8}, n, \Omega_{\text {other }}, h$, and $\Omega_{B}$.

\subsubsection{MCDM}

This is the simplest and the original CDM model; it is characterized by $b=1, n=1$, $\Omega_{\text {other }}=\Omega_{\text {cold }} \simeq 0.9, \Omega_{B} \sim 0.1$, and $h=0.5$. It is consistent with the COBE DMR data, which for $n=1$ imply $\sigma_{8}=1.2 \pm 0.2$, and intermediate-scale structure. However, it has too much power on small scales, quantified by a galaxy-pairwise velocity dispersion of about $1000 \mathrm{~km} \mathrm{~s}^{-1}$ compared to the observed $400 \mathrm{~km} \mathrm{~s}^{-1}$. A comparison of MCDM power spectrum with the observations is shown in Fig. 12.

Since MCDM is the simplest and most well motivated model perhaps inflationists should sit tight and wait for the data (or their interpretations) to change. After all, the disagreement is on small scales where the Universe is highly nonlinear and astrophysics can play an important role. 


\subsection{2 $\quad \mathrm{BCDM}$}

This is the CDM model with biasing, imposed to solve the problem of too much power on small scales. The parameters of this model are: $b \sim 1.5-2, n=1$, $\Omega_{\text {other }}=\Omega_{\text {cold }} \sim 0.9, \Omega_{B} \sim 0.1$, and $h=0.5$. This model is disfavor for two reasons: (1) The COBE DMR results imply $b=0.8 \pm 0.2$; and (2) (apparent) insufficient power on intermediate scales to account for peculiar velocities, the galaxy-galaxy angular correlation function, etc. However, one should keep in mind that the COBE DMR results are new and may still change, and that our knowledge of intermediate scales is the least secure. Perhaps the truth is somewhere in between MCDM and BCDM; both are well motivated. Shifting the MCDM power spectrum in Fig. 12 downward by a factor of $2-4$ corresponds to $\sigma_{8} \sim 0.5-0.7$.

\subsubsection{Tilt}

Tilted CDM $(\mathrm{TCDM})$ models are characterized by: $\sigma_{8} \sim 0.5, n \sim 0.8, \Omega_{\text {other }}=$ $\Omega_{\text {cold }} \sim 0.9, \Omega_{B} \sim 0.1$, and $h=0.5$ [100]. From the beginning it was realized that the inflationary perturbations were not precisely scale-invariant, typically with more power on large scales $(n<1)$ [72], and so tilted models too are well motivated. Relative to scale-invariant perturbations $(n=1)$ the density perturbation in a tilted model is

$$
\left(\frac{\delta \rho}{\rho}\right) \propto\left(\frac{\delta \rho}{\rho}\right)_{n=1} \lambda^{(1-n) / 2} .
$$

The COBE DMR result provides a normalization on very large scales, $\lambda \sim 10^{4} \mathrm{Mpc}$; relative to MCDM, the density perturbations on intermediate scales, $\lambda \sim 300 \mathrm{Mpc}$, are only a factor of about 1.4 smaller, while on small scales, $\lambda \sim 10 \mathrm{Mpc}$, they are about a factor of 2 smaller; see Fig. 13a.

If tilt is the truth, two kinds of inflationary potentials are singled out; exponential and low-order polynomial potentials [101]. Further, for exponential potentials, the contribution of gravity waves to the CBR anisotropy on large-angular scales is significant, which lowers the overall normalization of density perturbations further, by a factor of $(1+T / S)^{1 / 2}[12]$.

\subsubsection{Best-fit models}

These models address the problem of too much small-scale power by changing the transfer function. The models considered thus far are: cold dark matter with a cosmological constant $(\Lambda \mathrm{CDM}), n=1, \Omega_{B} \sim 0.05, \Omega_{\text {cold }} \sim 0.15, \Omega_{\Lambda} \sim 0.8$, and $h \sim 0.8$ [102]; and mixed dark matter (MDM) - "the neutrino cocktail-" $n=1$, $\Omega_{B} \sim 0.1, \Omega_{\text {cold }} \sim 0.6, h=0.5$, and $\Omega_{\text {hot }} \sim 0.3$, corresponding to a $7 \mathrm{eV}$ or so mass neutrino [103].

How is the transfer function changed? It is simplest to see in MDM; since part of the dark matter is in the form of neutrinos which freestream out of density fluctuations 
Figure 13: (a) TCDM vs. observation; (b) MDM/ $\mathrm{CDM}$ vs. observation. 
on small scales, perturbations on small scales are depressed (see Fig. 9), just what the doctor ordered. In $\Lambda \mathrm{CDM}$ the story is a little more complicated; the bend in the transfer function is set by the scale that crosses inside the horizon at matterradiation equality, $k_{\mathrm{EQ}} \sim 0.5\left(\Omega_{\text {matter }} h^{2}\right) \mathrm{Mpc}$, where $\Omega_{\text {matter }}=\Omega_{B}+\Omega_{\text {cold }} \simeq 0.2$. Relative to MCDM, $k_{\mathrm{EQ}}$ is a factor of two smaller, shifting the spectrum to smaller $k$ and decreasing power on small scales (see Fig. 9).

[The $\Lambda$ CDM model, which I once called "the best-fit Universe," has a number of other nice features. It automatically solves the $\Omega$ problem since $80 \%$ of the energy density is in vacuum energy which is uniformly distributed and thus does not "show up" in dynamical measurements of the mass density. It allows one to accommodate the higher values of the Hubble constant which are favored by many measurements. Likewise, MDM also address the $\Omega$ problem: there is not enough phase space in galaxies for neutrinos to account for halo masses; further, neutrinos probably move to fast to be captured even in clusters. This would explain why dynamical measurements of $\Omega_{0}$ based on galactic rotation curves or cluster virial masses do not lead to values of $\Omega_{0}$ close to unity.]

Because both $\Lambda$ CDM and MDM have less power on small scales than MCDM, when normalized to the COBE DMR result, they are a much better fit to the small scale data, and on large scales they are very similar to MCDM. They fit the present data very well; see Fig. 13b. One should, however, recall the words of Francis Crick (of DNA fame); loosely quoted,

A theory that agrees with all of the data at any given time is necessarily wrong, since at any given time some of the data are incorrect.

The weak point of the "best-fit models" is motivation; however, let me try to make the best case for each. $\Lambda$ CDM: A cosmological constant that contributes an energy density of about $10^{-46} \mathrm{GeV}^{4}$ would be very surprising. Since there is no physical mechanism known that explains why the present vacuum energy isn't of order $m_{\mathrm{Pl}}^{4}$ (perhaps with the help of supersymmetry only of order $G_{F}^{-2}$ ), one cannot rigorously say that $\rho_{\text {vac }} \simeq 10^{-46} \mathrm{GeV}^{4}$ is be fine-tuned in the technical sense. MDM: Cold dark matter has so many good features that it must be part of the truth; neutrinos existin three varieties - and the see-saw mechanism suggests nondegenerate masses in "the eV range" (meaning $10^{-6} \mathrm{eV}$ to tens of $\mathrm{eV}$ ), it could well be that one of the three neutrinos has a mass of order $10 \mathrm{eV}$.

Certainly neither case for motivation is strong. Why is the cosmological constant just today becoming dynamically significant (recall, $\rho_{\mathrm{vac}} / \rho_{\text {matter }} \propto R^{3}$ )? And if history is any guide, cosmologists beginning with Einstein have too often invoked a cosmological constant to solve their problems. For MDM, one has to posit two kinds of nonbaryonic dark matter that each contribute comparably to the energy density of the Universe. If nonbaryonic dark matter exists it is already puzzling that baryons and dark matter each contribute similar amounts to the mass density [104]. 


\subsection{The scorecard and future}

Whereas cosmologists used to talk about "the CDM model" and its "uniqueness" (words that were once used to describe the superstring), there is now a menu of CDM models. How do they stand, and which measurements can discriminate between them? In discussing their models, it is often said that theorists have many hands; on the one hand, on the other hand, on the other other hand and so on. Let me try my hand at it.

Occam's razor points to the simplest model, MCDM. Moreover, it was vindicated by $\mathrm{COBE}$ and only differs from the observational data by a factor of two or so on small scales where complicated astrophysics can be very important. Perhaps theorists should sit tight and wait. On the other hand (here we go), biasing at some level is likely to be a fact of life, arguing for BCDM; BCDM resolves the small-scale problems of CDM, but COBE indicates that $b \sim 1$. Maybe the truth is somewhere in between MCDM and BCDM; the COBE normalization could come down a bit, making $b \sim 1.3$ or so viable.

On the other other hand, deviation from scale-invariance was in the cards from the beginning, and so TCDM is well motivated too. Moreover, the tilt required points to a smaller class of inflationary models, exponential potentials and low-order polynomial potentials, which can be discriminated between by the size of their tensor perturbations.

On my final hands are the best-fit models, $\Lambda \mathrm{CDM}$ and MDM. They are not as well motivated, but agree better with the data at hand. Of the two, my first final hand has to go to MDM, and my second final hand to $\Lambda$ CDM.

There will be a variety of observations that can used to discriminate between the different CDM models; I will focus on CBR measurements on the $0.5^{\circ}-2^{\circ}$ scale, as there are several experiments with the sensitivity to probe CDM models which will be announcing results soon [105]. These experiments add roughly another order of magnitude to the range of scales probed by CBR anisotropy (recall, COBE probes $10^{\circ}$ to $\left.90^{\circ}\right)$. For reference, the MCDM prediction for this angular scale is $\delta T / T \sim$ $1-2 \times 10^{-5}$; the current upper limits are just above this level! Let me describe possible outcomes.

Scenario 1: The upper limits become detections. MCDM, $\Lambda \mathrm{CDM}$, and MDM are in; TCDM and BCDM are out.

Scenario 2: Detections are announced below the $10^{-5}$ level. BCDM and TCDM are in; the rest are out. If the detections are much below the $10^{-5}$ level, exponential potentials are strongly favored as in these tilted models much of the COBE signal is due to tensor perturbations whose contribution to CBR anisotropy falls dramatically around a few degrees [79, 12, 106].

Finally, let me mention a very different test, the value of the Hubble constant. Suppose all parties agree on the currently popular value $h=0.8$; all CDM models except $\Lambda$ CDM fall by the way side, based on the age of the Universe. Conversely, suppose that evidence for $h=0.5$ becomes overwhelming; $\Lambda \mathrm{CDM}$ is out. 


\section{Concluding Remarks}

Inflation is an extremely attractive cosmological paradigm; in spite - no, because - of its beauty it must be put to the ultimate test: confrontation with observation. In testing inflation one must focus on its robust predictions: In order of robustness, spatially flat Universe $\left(\Omega_{0}=1\right)$; very nearly scale-invariant spectrum of density perturbations; and nearly scale-invariant spectrum of gravitational waves. In addition, for first-order inflation there should be a "spike" in the stochastic background of gravitational waves of very significant energy density, $\Omega_{\mathrm{GW}} \sim 10^{-9}$ or so, at a frequency $f \sim 10^{4} \mathrm{~Hz}\left(\mathcal{M} / 10^{10} \mathrm{GeV}\right)$ [57]. It is also possible that fluctuations in other fields lead to primeval magnetic fields [107] or isocurvature perturbations (e.g., in axions or baryons) 69.

There are a variety of means of testing these predictions. For example, there are kinematic and dynamic techniques for measuring $\Omega_{0}$. The density perturbations lead to temperature fluctuations in the CBR. The tensor (gravity-wave) perturbations also lead to CBR anisotropies, or may be detected directly by the next generation of gravity wave detectors, Laser Interferometric Gravity-wave Observatories (LIGOs) [108.

From the "primary predictions," a series of secondary predictions follow. For example, since primordial nucleosynthesis restricts $\Omega_{B} \lesssim 0.1$, nonbaryonic dark matter is a necessity, and a host of experiments are under way to search for nonbaryonic dark matter [109]. In a flat, matter-dominated Universe $H_{0} t_{0}=\frac{2}{3}$ or $t_{0} \simeq 6.5 h^{-1} \mathrm{Gyr}$, which implies that $h$ must be greater than 0.65 to ensure that the Universe is older than 10 Gyr (the absolute minimum age that is consistent with other measures of the age of the Universe). The spectrum of density perturbations, together with the matter content, provide the initial data for the structure formation problem, leading to another test.

In the near term I believe that structure formation will provide the most powerful test of inflation and probe of inflationary models. On balance, the inflation-inspired CDM models are doing quite well so far compared to the alternatives: Texture and cosmic-string models required a high level of biasing $(b \sim 3)$ to be compatible with COBE DMR results; and PIB not only strongly violates the primordial nucleosynthesis constraint to $\Omega_{B}$ but also seems to be inconsistent with CBR anisotropy bounds on the scale of five degrees 86. Great efforts are being made to further test the CDM scenarios, and involve many different techniques, CBR anisotropy, red-shift surveys, peculiar velocity measurements, and so on. These observations not only have the power to falsify CDM, but could also reveal much about the inflationary potential: the value of the potential, its steepness, and the change in steepness, which in turn can used to learn about the underlying model. For example, suppose that density perturbations do deviate significantly from scale invariance, then two classes of models are ruled out - chaotic and new inflation - and two types of models are ruled in-exponential potentials (as found in extended inflation) or low-order polynomial potentials (as found in natural inflation). The ratio of tensor to scalar perturbations can further narrow the field: large tensor contribution to the CBR quadrupole 
points to exponential potentials and small-tensor contribution points to low-order polynomial potentials.

The moment of truth for inflation may be near!

This work was supported in part by the DOE (at Chicago and Fermilab) and by the NASA through grant NAGW-2381 (at Fermilab).

\section{References}

[1] For a textbook treatment of the standard cosmology see e.g., S. Weinberg, Gravitation and Cosmology (Wiley, NY, 1972); E.W. Kolb and M.S. Turner, The Early Universe (Addison-Wesley, Redwood City, CA, 1990).

[2] A. Sandage, Physica Scripta T43, 22 (1992).

[3] J. Mould et al., Astrophys. J., in press (1993).

[4] See e.g., M. Rowan-Robinson, The Cosmological Distance Ladder (Freeman, San Francisco, 1985).

[5] M. Fukugita, C.J. Hogan, and P.J.E. Peebles, Nature, in press (1993).

[6] J. Mather et al., Astrophys. J., in press (1993).

[7] P.J.E. Peebles, D.N. Schramm, E. Turner, and R. Kron, Nature 352, 769 (1991).

[8] H. Gush, M. Halpern, and E.H. Wishnow, Phys. Rev. Lett. 65, 537 (1990).

[9] G.F. Smoot et al., Astrophys. J. 396, L1 (1992); D.J. Fixsen et al., ibid, in press (1993).

[10] G.F. Smoot, in First Course in Current Topics in Astrofundamental Physics, eds. N. Sanchez and A. Zichichi (World Scientific, Singapore, 1992), p. 192.

[11] G.F. Smoot et al., Astrophys. J. 396, L1 (1992); E.L. Wright, ibid 396, L3 (1992).

[12] R. Davis et al., Phys. Rev. Lett. 69, 1856 (1992).

[13] E.L. Wright, ibid 396, L3 (1992). 
[14] S.S. Meyer, E.S. Cheng, and L.A. Page, Astrophys. J. 371, L1 (1991); K. Ganga, S.S. Meyer, E.S. Cheng, and L.A. Page, Astrophys. J., in press (1993).

[15] T.P. Walker et al., Astrophys. J. 376, 51 (1991).

[16] E.W. Kolb et al., Phys. Rev. Lett. 67, 533 (1991).

[17] For recent reviews of dark matter see e.g., M.S. Turner, Physica Scripta T36, 167 (1991); P.J.E. Peebles, Nature 321, 27 (1986); V. Trimble, Ann. Rev. Astron. Astrophys. 25, 425 (1987); J. Kormendy and G. Knapp, Dark Matter in the Universe (Reidel, Dordrecht, 1989); K. Ashman, Proc. Astron. Soc. Pac. 104, 1109 (1992); S. Faber and J. Gallagher, Ann. Rev. Astron. Astrophys. 17, 135 (1979).

[18] S. Faber and J. Gallagher, Ann. Rev. Astron. Astrophys. 17, 135 (1979).

[19] J.S. Mulchaey, D.S. Davis, R.F. Mushotzky, and D. Burstein, Astrophys. $J .$, in press (1993).

[20] M. Rowan-Robinson et al., Mon. Not. R. astr. Soc. 247, 1 (1990); N. Kaiser et al., ibid 252, 1 (1991); M. Strauss et al., Astrophys. J. 385, 444 (1992).

[21] E. Bertschinger and A. Dekel, Astrophys. J. 336, L5 (1989); A. Dekel et al., Astrophys. J., in press (1993); M. Strauss et al., ibid 397, 395 (1992).

[22] A. Sandage, Astrophys. J. 133, 355 (1961); Physica Scripta T43, 7 (1992); Refs. [1].

[23] E. Loh and E. Spillar, Astrophys. J. 307, L1 (1986); M. Fukugita et al., ibid 361, L1 (1990).

[24] See e.g., E.W. Kolb and M.S. Turner, Ann. Rev. Nucl. Part. Sci. 33, 645 (1983); A. Dolgov, Phys. Repts., in press (1993); A. Cohen, D. Kaplan, and A. Nelson, Ann. Rev. Nucl. Part. Sci., in press (1993).

[25] S. Weinberg, Gravitation and Cosmology (Wiley, NY, 1972).

[26] For a more complete pedagogical discussion of structure formation see e.g., Refs. [1]; P.J.E. Peebles, The Large-scale Structure of the Universe (Princeton Univ. Press, Princeton, 1980); G. Efstathiou, in The Physics of the Early Universe, eds. J.A. Peacock, A.F. Heavens, and A.T. Davies (Adam-Higler, Bristol, 1990).

[27] For a pedagogical discussion of CBR anisotropy see e.g., G. Efstathiou, in The Physics of the Early Universe, eds. J.A. Peacock, A.F. Heavens, and A.T. Davies (Adam-Higler, Bristol, 1990). Also see, J.R. Bond and 
G. Efstathiou, Mon. Not. R. astr. Soc. 226, 655 (1987); J.R. Bond et al., Phys. Rev. Lett. 66, 2179 (1991).

[28] R.K. Sachs and A.M. Wolfe, Astrophys. J. 147, 73 (1967).

[29] P.J.E. Peebles, Nature 327, 210 (1987); Astrophys. J. 315, L73 (1987);

R. Cen, J.P. Ostriker, and P.J.E. Peebles, ibid, in press (1993).

[30] See e.g., A. Vilenkin, Phys. Repts. 121, 263 (1985); A. Albrecht and A. Stebbins, Phys., Rev. Lett. 69, 2615 (1992); D. Bennett, A. Stebbins, and F. Bouchet, Astrophys. J. 399, L5 (1992).

[31] See e.g., N. Turok, Phys. Rev. Lett. 63, 2652 (1989); A. Gooding, D. Spergel, and N. Turok, Astrophys. J. 372, L5 (1991).

[32] J. Fry, C.T. Hill, and D.N. Schramm, Comments on Nucl. Part. Phys. 19, 25 (1989); A. Gupta et al., Phys. Rev. D 45, 441 (1992).

[33] See e.g., C.W. Misner, Astrophys. J. 151, 431 (1968); R. Penrose, in General Relativity: An Einstein Centenary Survey, eds. S.W. Hawking and W. Israel (Cambridge Univ. Press, Cambridge, 1979); R.H. Dicke and P.J.E. Peebles, ibid.

[34] J. Preskill, Ann. Rev. Nucl. Part. Sci. 34, 461 (1984).

[35] C.B. Collins and S.W. Hawking, Astrophys. J. 180, 317 (1973).

[36] A.H. Guth, Phys. Rev. D 23, 347 (1981).

[37] Y. Hu, M.S. Turner, and E.J. Weinberg, Phys. Rev. Lett., in press (1993).

[38] S. Coleman, Phys. Rev. D 15, 2929 (1977); S. Coleman and R. De Luccia, ibid 21, 3305 (1980).

[39] R. Watkins and L. Widrow, Nucl. Phys. B 374, 446 (1992); S.W. Hawking, J. Stewart, and I. Moss, Phys. Rev. D 26, 2681 (1982).

[40] A.D. Linde, Phys. Lett. B 108, 389 (1982).

[41] A. Albrecht and P.J. Steinhardt, Phys. Rev. Lett. 48, 1220 (1982).

[42] A. Albrecht et al., Phys. Rev. Lett. 48, 1437 (1982); L. Abbott and M. Wise, Phys. Lett. B 117, 29 (1982); A.D. Linde and A. Dolgov, ibid 116, 329 (1982).

[43] For a review of first-order inflation see e.g., E.W. Kolb, Physica Scripta T36, 199 (1991).

[44] D. La and P.J. Steinhardt, Phys. Rev. Lett. 62, 376 (1989). 
[45] F. Adams and K. Freese, Phys. Rev. D 43, 353 (1991).

[46] E.W. Kolb, D. Salopek, and M.S. Turner, Phys. Rev. D 42, 3925 (1990).

[47] E.J. Weinberg, Phys. Rev. D 40, 3950 (1989); M.S. Turner, E.J. Weinberg, and L. Widrow, ibid 46, 2384 (1992).

[48] Q. Shafi and A. Vilenkin, Phys. Rev. Lett. 52, 691 (1984).

[49] S.-Y. Pi, Phys. Rev. Lett. 52, 1725 (1984).

[50] L. Knox and M.S. Turner, Phys. Rev. Lett. 70, 371 (1993).

[51] K.A. Olive, Phys. Repts. 190, 307 (1990).

[52] R. Holman, P. Ramond, and G.G. Ross, Phys. Lett. B 137, 343 (1984).

[53] K. Freese, J. Frieman, and A. Olinto, Phys. Rev. Lett. 65, 3233 (1990).

[54] A.D. Linde, Phys. Lett. B 129, 177 (1983).

[55] Q. Shafi and C. Wetterich, Phys. Lett. B 129, 387 (1983); ibid 152, 51 (1985).

[56] A.A. Starobinski, Phys. Lett. B 91, 99 (1980); M.B. Mijic, M.S. Morris, and W.-M. Suen, Phys. Rev. D 34, 2934 (1986).

[57] M.S. Turner and F. Wilczek, Phys. Rev. Lett. 65, 3080 (1990); A. Kosowsky, M.S. Turner, and R. Watkins ibid 69, 2026 (1992).

[58] R.D. Reasenberg et al., Astrophys. J. 234, L219 (1979).

[59] M.S. Turner and L. Widrow, Phys. Rev. Lett. 57, 2237 (1986).

[60] R.M. Wald, Phys. Rev. D 28, 2118 (1983); L. Jensen and J. Stein-Schabes, ibid 34, 931 (1986).

[61] L. Jensen and J. Stein-Schabes, Phys. Rev. D 35, 1146 (1987).

[62] A.A. Starobinskii, JETP Lett. 37, 66 (1983).

[63] J.A. Frieman and M.S. Turner, Phys. Rev. D 30, 265 (1984).

[64] D. Goldwirth and T. Piran, Phys. Repts. 214, 223 (1992).

[65] See e.g., A.D. Linde, Inflation and Quantum Cosmology (Academic Press, San Diego, CA, 1990). 
[66] A.H. Guth and S.-Y. Pi, Phys. Rev. Lett. 49, 1110 (1982); A.A. Starobinskii, Phys. Lett. B 117, 175 (1982); S.W. Hawking, ibid 115, 295 (1982); J.M. Bardeen, P.J. Steinhardt, and M.S. Turner, Phys. Rev. D 28, 679 (1983).

[67] V.A. Rubakov, M. Sazhin, and A. Veryaskin, Phys. Lett. B 115, 189 (1982); R. Fabbri and M. Pollock, ibid 125, 445 (1983); L. Abbott and M. Wise, Nucl. Phys. B 244, 541 (1984); B. Allen, Phys. Rev. D 37, 2078 (1988).

[68] At first sight, first-order inflation might seem very different from slowrollover inflation, as reheating occurs through the nucleation of percolation of true-vacuum bubbles. However, such models can be recast as slow-rollover inflation by means of a conformal transformation, and the analysis of metric perturbations proceeds as in slow rollover inflation. See e.g., E.W. Kolb, D. Salopek, and M.S. Turner, Phys. Rev. D 42, 3925 (1990).

[69] See e.g., A.D. Linde, Phys. Lett. B 158, 375 (1985); D. Seckel and M.S. Turner, Phys. Rev. D 32, 3178 (1985); M.S. Turner, A. Cohen, and D. Kaplan, Phys. Lett. B 216, 20 (1989).

[70] E.W. Kolb and M.S. Turner, The Early Universe (Addison-Wesley, Redwood City, CA, 1990), Ch. 8.

[71] E.R. Harrison, Phys. Rev. D 1, 2726 (1970); Ya.B. Zel'dovich, Mon. Not. R. astr. Soc. 160, 1p (1972).

[72] P.J. Steinhardt and M.S. Turner, Phys. Rev. D 29, 2162 (1984).

[73] The material presented in this Section is a summary of work completed during this school and will be published elsewhere, M.S. Turner, Phys. Rev. D, in press (1993).

[74] J.M. Bardeen et al., Astrophys. J. 304, 15 (1986).

[75] D.H. Lyth and E.D. Stewart, Phys. Lett. B 274, 168 (1992); E.D. Stewart and D.H. Lyth, ibid, in press (1993).

[76] L. Abbott and M. Wise, Nucl. Phys. B 244, 541 (1984).

[77] M. White, Phys. Rev. D 46, 4198 (1992).

[78] M.S. Turner, Phys. Rev. D, in press (1993).

[79] M.S. Turner and J.E. Lidsey, Phys. Rev. D, in press (1993). 
[80] L. Abbott and M. Wise, Nucl. Phys. B 244, 541 (1984); F. Lucchin and S. Mattarese, Phys. Rev. D 32, 1316 (1985); R. Fabbri, F. Lucchin, and S. Mattarese, Phys. Lett. B 166, 49 (1986).

[81] V. Belinsky, L. Grishchuk, I. Khalatanikov, and Ya.B. Zel'dovich, Phys. Lett. B 155, 232 (1985); L. Jensen, unpublished (1985).

[82] A.A. Starobinskii, Sov. Astron. 11, 133 (1985).

[83] A.D. Linde, Phys. Lett. B 108, 389 (1982); A. Albrecht and P.J. Steinhardt, Phys. Rev. Lett. 48, 1220 (1982).

[84] S.D.M. White, C. Frenk, and M. Davis, Astrophys. J. 274, L1 (1983); ibid 287, 1 (1983); J. Centrella and A. Melott, Nature 305, 196 (1982).

[85] J.P. Ostriker, Ann. Rev. Astron. Astrophys. 31, in press (1993).

[86] L. Knox and M.S. Turner, Astrophys. J., in press (1993).

[87] V. De Lapparent, M. Geller, and J. Huchra, Astrophys. J. 302, L1 (1986); ibid 332, 44 (1988); M. Geller and J. Huchra, Science 246, 897 (1989).

[88] N. Bahcall, Ann. Rev. Astron. Astrophys. 26, 631 (1988).

[89] M. Davis et al., Astrophys. J. 292, 371 (1985).

[90] T. Broadhurst et al., Nature 343, 726 (1990).

[91] S.J. Maddox et al., Mon. Not. R. astr. Soc. 242, 43p (1990).

[92] A. Dressler et al., Astrophys. J. 313, L37 (1987); E. Bertschinger et al., ibid 364, 370 (1990) and references therein.

[93] K. Fisher et al., Astrophys. J. 389, 188 (1992); also see, M.S. Vogeley et al., ibid 391, L5 (1992); W. Saunders et al., Nature 349, 42 (1991).

[94] J. Loveday et al., Astrophys. J. 390, 338 (1992); ibid 400, L43 (1992).

[95] The Sloan Digital Sky Survey is a collaboration between The University of Chicago, Fermilab, Johns Hopkins University, Princeton University and the Institute for Advanced Study.

[96] N. Kaiser, Astrophys. J. 284, L9 (1984); in Inner Space/Outer Space, eds. E.W. Kolb et al. (Univ. of Chicago Press, Chicago, 1986), p. 258.

[97] G. Efstathiou, J.R. Bond, and S.D.M. White, Mon. Not. R. astr. Soc. 258, 1p (1992). 
[98] R.Y. Cen and J.P. Ostriker, Astrophys. J. 393, 22 (1992); A.E. Evrard, F.J. Summers, and M. Davis, Astrophys. J., in press (1993); E. Bertschinger and J. Gelb, Computers in Physics 5, 164 (1991); J. Gelb and E. Bertschinger, Astrophys. J., in press (1993); N. Katz, L. Hernquist, and D. Weinberg, Astrophys. J. 399, L109 (1992); G. Evrard, Mon. Not. R. astr. Soc. 235, 911 (1988); C. Park and J.R. Gott ibid 249, 288 (1991); C. Park, ibid 242, 59p (1990); C. Frenk et al., Astrophys. J. 351, $10(1990)$.

[99] For a synopsis of structure formation in the CDM scenario see e.g., G.R. Blumenthal et al., Nature 311, 517 (1984).

[100] See e.g., J.P. Ostriker, Ann. Rev. Astron. Astrophys. 31, in press (1993); F. Adams et al., Phys. Rev. D 47, 426 (1993); J. Gelb et al., Astrophys. J. 403, L5 (1993); R. Cen and J.P. Ostriker, ibid, in press (1993).

[101] R. Davis et al., Phys. Rev. Lett. 69, 1856 (1992); F. Lucchin, S. Mattarese, and S. Mollerach, Astrophys. J. 401, L49 (1992); D. Salopek, Phys. Rev. Lett. 69, 3602 (1992); A. Liddle and D. Lyth, Phys. Lett. B 291, 391 (1992); J.E. Lidsey and P. Coles, Queen Mary College preprint (1992); A. Dolgov and J. Silk, unpublished (1992); T. Souradeep and V. Sahni, Mod. Phys. Lett. A 7, 3541 (1992).

[102] M.S. Turner, G. Steigman, and L. Krauss, Phys. Rev. Lett. 52, 2090 (1984); M.S. Turner, Physica Scripta T36, 167 (1991); P.J.E. Peebles, Astrophys. J. 284, 439 (1984); G. Efstathiou et al., Nature 348, 705 (1990).

[103] Q. Shafi and F. Stecker, Phys. Rev. Lett. 53, 1292 (1984); S. Achilli, F. Occhionero, and R. Scaramella, Astrophys. J. 299, 577 (1985); S. Ikeuchi, C. Norman, and Y. Zahn, Astrophys. J. 324, 33 (1988); A. van Dalen and R.K. Schaefer, Astrophys. J. 398, 33 (1992); M. Davis, F. Summers, and D. Schlegel, Nature 359, 393 (1992); J. Holtzman and J.A. Primack, Astrophys. J., in press (1993); A. Klypin et al., Astrophys. J., in press (1993); D. Pogosyan and A.A. Starobinsky, DAMTP/IOA/MRAO preprint (1993).

[104] M.S. Turner and B.J. Carr, Mod. Phys. Lett. A 2, 1 (1987).

[105] T. Gaier et al., Astrophys. J. 398, L1 (1992); D.C. Alsop et al., ibid 387, 146 (1992); A.C.S. Readhead et al., ibid 346, 566 (1989); P. De Bernardis et al., ibid 396, L57 (1992); R.A. Watson et al., Nature 357, 660 (1992); S.S. Meyer, E.S. Cheng, and L.A. Page, Astrophys. J. 371, L1 (1991); J.O. Gundersen et al., ibid, in press (1993); P.R. Meinhold et al., ibid, in press (1993). 
[106] J.R. Bond, R. Crittenden, R. Davis, G. Efstathiou, and P.J. Steinhardt, Phys. Rev. Lett., in press (1993).

[107] M.S. Turner and L.M. Widrow, Phys. Rev. D 37, 2743 (1988); B. Ratra, Astrophys. J. 391, L1 (1992).

[108] A. Abramovici et al., Science 256, 325 (1992); K.S. Thorne, in 300 Years of Gravitation, eds. S.W. Hawking and W. Israel (Cambridge Univ. Press, Cambridge, 1987), p. 330.

[109] See e.g., J.R. Primack, D. Seckel, and B. Sadoulet, Ann. Rev. Nucl. Part. Sci. 38, 751 (1988); D.O. Caldwell, Mod. Phys. Lett. A 5, 1543 (1990); P.F. Smith and J.D. Lewin, Phys. Repts. 187, 203 (1990). 